\title{
Minimal energy for the traveling waves of the Landau-Lifshitz equation
}

\author{
André de Laire \\ Laboratoire Paul Painlevé, Université de Lille 1 \\ 59655 Villeneuve d'Ascq Cedex, France \\ andre.de-laire@math.univ-lille1.fr
}

\begin{abstract}
We consider nontrivial finite energy traveling waves for the Landau-Lifshitz equation with easyplane anisotropy. Our main result is the existence of a minimal energy for these traveling waves, in dimensions two, three and four. The proof relies on a priori estimates related with the theory of harmonic maps and the connection of the Landau-Lifshitz equation with the kernels appearing in the Gross-Pitaevskii equation.
\end{abstract}

2010 Mathematics Subject Classification: 35J60, 35Q51, 37K40, 35B65, 58E20, 82D55

Keywords and phrases: Landau-Lifshitz equation, Traveling waves, Minimal energy, Harmonic maps, Asymptotic behavior

\section{Introduction}

In this work we consider the Landau-Lifshitz equation

$$
\partial_{t} m+m \times\left(\Delta m+\lambda m_{3} e_{3}\right)=0, \quad m(t, x) \in \mathbb{S}^{2}, t \in \mathbb{R}, x \in \mathbb{R}^{N},
$$

where $e_{3}=(0,0,1), \lambda \in \mathbb{R}$ and $m=\left(m_{1}, m_{2}, m_{3}\right)$. This equation was originally introduced by L. Landau and E. Lifshitz in 32 to describe the dynamics of magnetization in ferromagnetic materials. Here the parameter $\lambda$ takes into account the anisotropy of such material. More precisely, the value $\lambda=0$ corresponds to the isotropic case, meanwhile $\lambda>0$ and $\lambda<0$ correspond to materials with an easy-axis and an easy-plane anisotropy, respectively (see [29, 25]).

The isotropic case $\lambda=0$ recovers the Schödinger map equation, which has been intensively studied due to its applications in several areas of physics and mathematics (see [17, 38]). For $\lambda>0$, the existence of solitary waves periodic in time have been established in [20, 41]. Moreover, $\mathrm{Pu}$ and Guo [42] showed that $\lambda \neq 0$ is a necessary condition to the existence of these types of solutions.

In this paper we are interested in the case of easy-plane anisotropy $\lambda<0$. By a scaling argument we can suppose from now on that $\lambda=-1$. Then the energy of (1.1) is given by

$$
E(m)=\int_{\mathbb{R}^{N}} e(m) d x \equiv \frac{1}{2} \int_{\mathbb{R}^{N}}\left(|\nabla m|^{2}+m_{3}^{2}\right) d x,
$$

that it is formally conserved due to the Hamiltonian structure of (1.1). If $m$ is smooth, by differentiating twice the condition $|m(t, x)|^{2}=1$ we obtain $m \cdot \Delta m=-|\nabla m|^{2}$, so that taking cross product of $m$ and (1.1), we can recast (1.1) as

$$
m \times \partial_{t} m=\Delta m+|\nabla m|^{2} m-\left(m_{3} e_{3}-m_{3}^{2} m\right) .
$$

Using formal developments and numerical simulations, Papanicolaou and Spathis 40 found in dimensions $N \in\{2,3\}$ nonconstant finite energy traveling waves of (1.2), propagating with speed $c \in(0,1)$ along the $x_{1}$-axis, i.e. of the form

$$
m_{c}(x, t)=u\left(x_{1}-c t, x_{2}, \ldots, x_{N}\right) .
$$


By substituting $m_{c}$ in (1.2), the profile $u$ satisfies

$$
-\Delta u=|\nabla u|^{2} u+u_{3}^{2} u-u_{3} e_{3}+c u \times \partial_{1} u .
$$

Notice that if $u$ satisfies $\left(\mathrm{TW}_{c}\right)$ with speed $c$, so does $-u$ with speed $-c$, therefore we can assume that $c \geq 0$. Also, we see that any constant in $\mathbb{S}^{1} \times\{0\}$ satisfies $\left(\mathrm{TW}_{c}\right)$, so that we refer to them as the trivial solutions. Since we are interested in finite energy solutions, the natural energy space to work in is

$$
\mathcal{E}\left(\mathbb{R}^{N}\right)=\left\{v \in L_{\text {loc }}^{1}\left(\mathbb{R}^{N} ; \mathbb{R}^{3}\right): \nabla v \in L^{2}\left(\mathbb{R}^{N}\right), v_{3} \in L^{2}\left(\mathbb{R}^{N}\right),|v|=1 \text { a.e. on } \mathbb{R}^{N}\right\} .
$$

\subsection{The minimal energy}

Our main theorem is in the same spirit as the result proved by the author for the Gross-Pitaevskii equation in [9] (see also 2]). Precisely, we show the existence of a minimal value for the energy for the nontrivial traveling waves.

Theorem 1.1. Let $N \in\{2,3,4\}$. There exists a universal constant $\mu>0$ such that if $u \in \mathcal{E}\left(\mathbb{R}^{N}\right)$ is a nontrivial solution of $\left[\mathrm{TW}_{c}\right.$ with $c \in(0,1]$, satisfying in addition that $u$ is uniformly continuous if $N \in\{3,4\}$, then

$$
E(u) \geq \mu .
$$

As noticed in 21] in dimension two, there is no smooth static solution of $\left(\mathrm{TW}_{c}\right)$, i.e. with speed $c=0$. More generally, we obtain the following result for static waves.

Proposition 1.2. Let $N \geq 2$. Assume that $u \in \mathcal{E}\left(\mathbb{R}^{N}\right)$ is a solution of $\left(\mathrm{TW}_{c}\right.$ with $c=0$. Suppose also that $u$ is uniformly continuous if $N \geq 3$. Then $u$ is a trivial solution.

Theorem 1.1] shows that there are no small energy traveling wave solutions in dimensions two, three and four (assuming that they are uniformly continuous in dimensions three and four). This opens the door to have a scattering theory for equation (1.1) with $\lambda=-1$, similarly to the theory developed for the Gross-Pitaevskii equation by Gustafson, Nakanishi and Tsai [18, 19].

The one-dimensional case is different. If $N=1,\left(\overline{T W_{c}}\right)$ is completely integrable and we can compute the solutions in $\mathcal{E}(\mathbb{R})$ explicitly. More precisely,

Proposition 1.3. Let $N=1, c \geq 0$ and $u \in \mathcal{E}(\mathbb{R})$ be solution of $\left(\overline{T_{c}}\right.$.

(i) If $c \geq 1$, then $u$ is a trivial solution.

(ii) If $0 \leq c<1$ and $u$ is nontrivial, then, up to invariances, $u$ is given by

$$
u_{1}=c \operatorname{sech}\left(\sqrt{1-c^{2}} x\right), u_{2}=\tanh \left(\sqrt{1-c^{2}} x\right), u_{3}=\sqrt{1-c^{2}} \operatorname{sech}\left(\sqrt{1-c^{2}} x\right) .
$$

Moreover, if $0<c<1$,

$$
E(u)=2 \sqrt{1-c^{2}} \quad \text { and } \quad E(p(u))=2 \sin (p(u) / 2),
$$

where $p(u)$ denotes the momentum of $u$.

We notice that equation $\left(\mathrm{TW}_{c}\right)$ is invariant under translations and under the action of $\mathbb{S}^{1}$ by a rotation around the $e_{3}$-axis, that is if $u=\left(u_{1}, u_{2}, u_{3}\right)$ is a solution of $\left(\mathrm{TW}_{c}\right)$, so is

$$
\left(u_{1} \cos (\varphi)-u_{2} \sin (\varphi), u_{1} \sin (\varphi)+u_{2} \cos (\varphi), u_{3}\right),
$$

for any $\varphi \in \mathbb{R}$. Also, if $u=\left(u_{1}, u_{2}, u_{3}\right)(x)$ is a solution, so is $u=\left(u_{1}, u_{2},-u_{3}\right)(-x)$. These are the invariances that we refer to in Proposition 1.3 .

We provide the proof of Proposition 1.3 in Section [6, as well as the precise definition of momentum. The relation $E=2 \sin (p / 2)$ is showed in Figure 1. In particular we note that there are solutions of small energy, but there is a maximum value for the energy and the momentum. We also remark that Proposition 1.3 provides a solution with $c=0$, meanwhile Proposition 1.2 states that this is not possible in the case $N \geq 2$. 


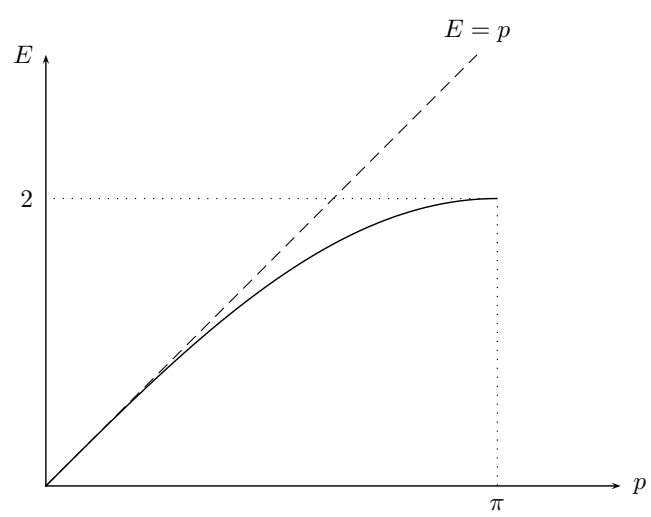

Figure 1: Curve of energy $E$ as a function of the momentum $p$ in the one-dimensional case.

\subsection{From Gross-Pitaevskii to Landau-Lifshitz}

The results of this paper have been motivated by the numerical simulations in [40], where the authors determine a branch of nontrivial solutions of $\left(\mathrm{TW}_{c}\right)$, axisymmetric around the $x_{1}$-axis, for any speed $c \in(0,1)$ in dimensions two and three. They also conjecture that there is no nontrivial finite energy solution of $\left(\mathrm{TW}_{c}\right)$ for $c \geq 1$. For $c$ small, the existence of these traveling waves has been proved rigorously by Lin and Wei [33. The branch of solutions is depicted in Figure2. We see that the curve has a nonzero minimum, which represents the minimal energy in Theorem 1.1

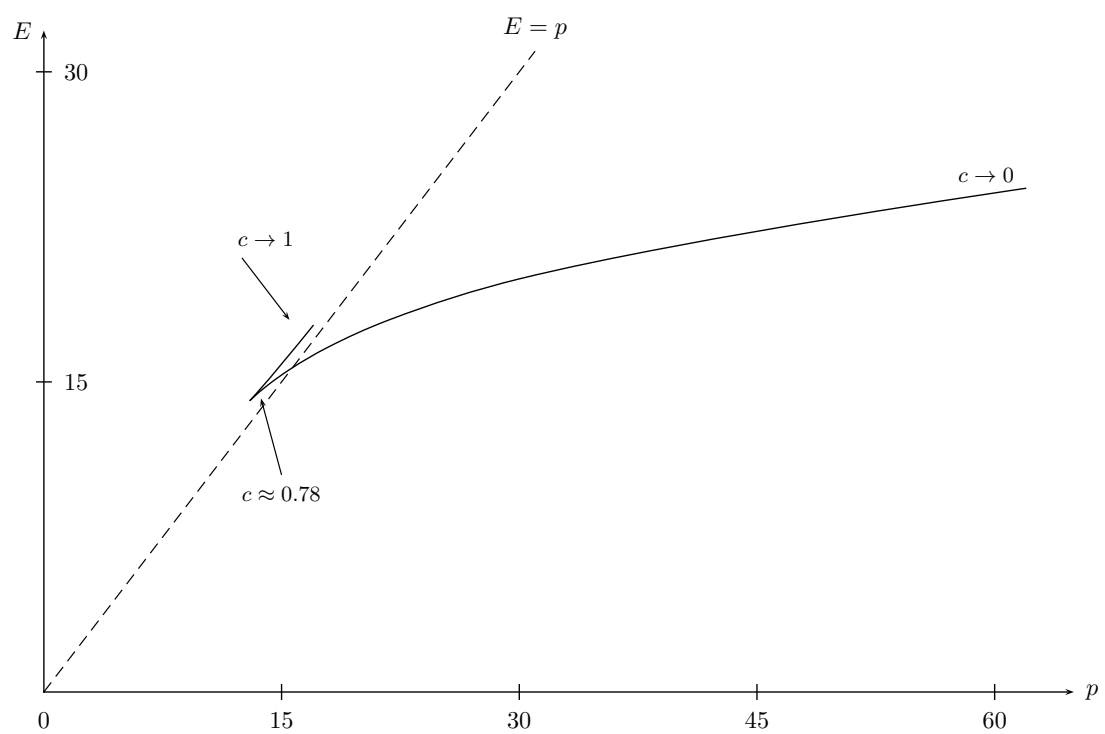

Figure 2: Curve of energy $E$ as a function of the momentum $p$ in the two-dimensional case.

Some properties of the solutions found in [40] are very similar to those of the traveling waves for the Gross-Pitaevskii equation obtained numerically by Jones, Putterman and Roberts [27, 26] and studied rigourously in [1, 2, 35. In fact, if $u$ is a solution of $\left(\mathrm{TW}_{c}\right)$, the stereographic variable

$$
\psi=\frac{u_{1}+i u_{2}}{1+u_{3}}
$$

satisfies

$$
\Delta \psi+\frac{1-|\psi|^{2}}{1+|\psi|^{2}} \psi-i c \partial_{1} \psi=\frac{2 \bar{\psi}}{1+|\psi|^{2}}(\nabla \psi)^{2},
$$

that seems like a perturbed equation for the traveling waves for the Gross-Pitaevskii equation, namely

$$
\Delta \Psi+\left(1-|\Psi|^{2}\right) \Psi-i c \partial_{1} \Psi=0 .
$$


However, other properties of the solutions are very different. For instance, the energy-momentum curve for the Gross-Pitaevskii equation tends to zero as the momentum goes to zero in the two-dimensional case, but there exists a minimal energy if $N \geq 3$ (see [2, 9]).

From a mathematical point of view, (1.4) is a quasilinear Schrödinger equation meanwhile (1.5) is a semilinear Schrödinger equation. Therefore, it is not clear how to relate both equations. One of the purposes of this paper is to clarify this connection, to show how to exploit the similarities between (1.4) and (1.5), and how to deal with the extra difficulties of equation (TW $)$. In particular, we will discuss the regularity of the solutions of $\left(\mathrm{TW}_{c}\right)$, some a priori bounds and their asymptotic behavior as $|x| \rightarrow \infty$.

\subsection{Sketch of the proof of Theorem 1.1}

The starting point of our analysis is that for any solution $u \in \mathcal{E}\left(\mathbb{R}^{N}\right)$ of $\left(\mathrm{TW}_{c}\right)$, there exists $R \equiv R(u)$ such that we have the lifting

$$
\check{u} \equiv u_{1}+i u_{2}=\varrho e^{i \theta}, \quad \text { on } B(0, R)^{c},
$$

where $\varrho \equiv \sqrt{u_{1}^{2}+u_{2}^{2}}=\sqrt{1-u_{3}^{2}}$ and $\varrho, \theta \in \dot{H}^{1}\left(B(0, R)^{c}\right)$ (see Lemma 2.4). Let $\chi \in C^{\infty}\left(\mathbb{R}^{N}\right)$ be such that $|\chi| \leq 1, \chi=0$ on $B(0,2 R)$ and $\chi=1$ on $B(0,3 R)^{c}$, if $R>0$. In the case that $R=0$, we let $\chi=1$ on $\mathbb{R}^{N}$. In this way, we can assume that the function $\chi \theta$ and

$$
G=\left(G_{1}, \ldots, G_{N}\right) \equiv u_{1} \nabla u_{2}-u_{2} \nabla u_{1}-\nabla(\chi \theta),
$$

is well-defined on $\mathbb{R}^{N}$. For $u=\left(u_{1}, u_{2}, u_{3}\right)$, equation $\mathrm{TW}_{c}$ reads

$$
\begin{aligned}
& -\Delta u_{1}=2 e(u) u_{1}+c\left(u_{2} \partial_{1} u_{3}-u_{3} \partial_{1} u_{2}\right), \\
& -\Delta u_{2}=2 e(u) u_{2}+c\left(u_{3} \partial_{1} u_{1}-u_{1} \partial_{1} u_{3}\right), \\
& -\Delta u_{3}=2 e(u) u_{3}-u_{3}+c\left(u_{1} \partial_{1} u_{2}-u_{2} \partial_{1} u_{1}\right) .
\end{aligned}
$$

Then, using (1.8) and (1.9),

$$
\begin{aligned}
\operatorname{div}(G) & =u_{1} \Delta u_{2}-u_{2} \Delta u_{1}-\Delta(\chi \theta) \\
& =c\left(\partial_{1} u_{3}-u_{3} u \cdot \partial_{1} u\right)-\Delta(\chi \theta) \\
& =c \partial_{1} u_{3}-\Delta(\chi \theta)
\end{aligned}
$$

where we used the fact that $u \cdot \partial_{1} u=0$. By combining with (1.10), we obtain

$$
\Delta^{2} u_{3}-\Delta u_{3}+c^{2} \partial_{11}^{2} u_{3}=-\Delta F+c \partial_{1}(\operatorname{div} G), \quad \text { on } \mathbb{R}^{N} .
$$

At this point we remark that the differential operator

$$
\Delta^{2}-\Delta+c^{2} \partial_{11}^{2}
$$

is elliptic if and only if $c \leq 1$, which shows that $c=1$ is a critical value for the equation (TW $\mathrm{TW}_{c}$.

Taking Fourier transform in (1.12), we get

$$
\left(|\xi|^{4}+|\xi|^{2}-c^{2} \xi_{1}^{2}\right) \widehat{u}_{3}(\xi)=|\xi|^{2} \widehat{F}(\xi)-c \sum_{j=1}^{N} \xi_{1} \xi_{j} \widehat{G}_{j}(\xi),
$$

and hence

$$
\widehat{u}_{3}(\xi)=L_{c}(\xi)\left(\widehat{F}(\xi)-c \sum_{j=1}^{N} c \frac{\xi_{1} \xi_{j}}{|\xi|^{2}} \widehat{G}_{j}(\xi)\right)
$$

where

$$
L_{c}(\xi)=\frac{|\xi|^{2}}{|\xi|^{4}+|\xi|^{2}-c^{2} \xi_{1}^{2}} .
$$

Equivalently, we can write (1.14) as the convolution equation

$$
u_{3}=\mathcal{L}_{c} * F-c \sum_{j=1}^{N} \mathcal{L}_{c, j} * G_{j}
$$


where $\widehat{\mathcal{L}}_{c}=L_{c}$ and

$$
\widehat{\mathcal{L}}_{c, j}=\frac{\xi_{1} \xi_{j}}{|\xi|^{4}+|\xi|^{2}-c^{2} \xi_{1}^{2}} .
$$

Similarly, from (1.11) and (1.14), for $j \in\{1, \ldots, N\}$,

$$
\partial_{j}(\chi \theta)=c \mathcal{L}_{c, j} * F-c^{2} \sum_{k=1}^{N} \mathcal{T}_{c, j, k} * G_{k}-\sum_{k=1}^{N} \mathcal{R}_{j, k} * G_{k},
$$

where

$$
\widehat{\mathcal{T}}_{c, j, k}=\frac{\xi_{1} \xi_{j} \xi_{k}}{|\xi|^{2}\left(|\xi|^{4}+|\xi|^{2}-c^{2} \xi_{1}^{2}\right)} \quad \text { and } \quad \widehat{\mathcal{R}}_{j, k}=\frac{\xi_{j} \xi_{k}}{|\xi|^{2}}
$$

for all $j, k \in\{1, \ldots, N\}$.

The kernels $\mathcal{L}_{c}, \mathcal{L}_{c, j}, \mathcal{R}_{j, k}$ and $\mathcal{T}_{c, j, k}$ are the same as those appearing in the Gross-Pitaevskii equation (1.5). As a consequence, all the properties valid for the (1.5) depending only in the structure of these kernels, can be transfer to the Landau-Lifshitz equation. For instance, the asymptotic behavior theory developed in [14, 16, 4, 8, can be applied, after proving some algebraic decay of the solutions. We provide a precise statement in Theorem 1.6] at the end of this introduction.

Roughly speaking, the principle used to find a minimal energy for the traveling waves of (1.5) in [2, 9, written in the context of the equation $\left(\mathrm{TW}_{c}\right)$, is that on one hand a convolution equation such as (1.15) should imply that

$$
\left\|u_{3}\right\|_{L^{p}} \leq C\left(\|u\|_{W^{k, q}}\right) E(u)^{\gamma},
$$

for some $q, k \in \mathbb{N}$, and $\gamma>0$. On the other hand, using (1.8), (1.9), (1.10) and integrating by parts one should get an a priori bound for the energy of the form:

$$
E(u) \leq C\left(\|u\|_{W^{\ell, r}}\right)\left\|u_{3}\right\|_{L^{p}}^{\delta},
$$

for some $\ell, r \in \mathbb{N}$, and $\delta>0$. By putting together (E1) and (E2),

$$
E(u) \leq C\left(\|u\|_{W^{\ell, r}}\right) C\left(\|u\|_{W^{k, p}}\right)^{\delta} E(u)^{\gamma \delta} .
$$

Notice that we can assume that $E(u)>0$ because $u$ is not constant. If $\gamma \delta>1$ and if

$$
C\left(\|u\|_{W^{\ell, r}}\right) C\left(\|u\|_{W^{k, p}}\right)^{\delta} \leq M,
$$

for some constant $M$ independent of $u$ and $c$, we can conclude that

$$
\frac{1}{M^{1 /(\gamma \delta-1)}} \leq E(u)
$$

so that we have the existence of a minimal energy.

In conclusion, we have reduced the proof of Theorem 1.1 to the proof of the estimates (E1), (E2) and (E3), for some $\gamma, \delta>0$ such that $\gamma \delta>1$.

\subsubsection{Estimate $(\mathrm{E} 3)$ and regularity of traveling waves}

Let us consider the quasilinear elliptic system,

$$
\Delta u=f(x, u, \nabla u), \quad \text { in } \Omega,
$$

where $\Omega$ is a smooth domain and $f$ is a smooth function with quadratic growth

$$
|f(x, z, p)| \leq A+B|p|^{2} .
$$

We notice that the square-gradient term prevents us from invoking the usual elliptic regularity estimates. However, well-known regularity results imply that every continuous solution in $H^{1}(\Omega)$ belongs to $H_{\text {loc }}^{2,2}(\Omega) \cap C_{\text {loc }}^{0, \alpha}(\Omega)$ (see [12, 31, [5, 28]), but in general we do not have nice a priori estimates such as in 
the $L^{p}$-regularity theory because the $H_{\mathrm{loc}}^{2,2}(\Omega)$-norm depends on the modulus of continuity of the $u$. To exemplify this point, let us consider the harmonic map equation

$$
-\Delta v=|\nabla v|^{2} v, \quad \text { in } \Omega, v \in \mathbb{S}^{2} .
$$

Let $\Omega=\mathbb{R}^{N}, N \geq 2$, and assume that there exists $C_{1}, C_{2}, \alpha>0$ such that

$$
\|\nabla v\|_{L^{\infty}\left(\mathbb{R}^{N}\right)} \leq C_{1}+C_{2}\|\nabla v\|_{L^{2}\left(\mathbb{R}^{N}\right)}^{\alpha} .
$$

Note that $\|\nabla v\|_{L^{2}\left(\mathbb{R}^{N}\right)}^{2}$ is the energy associated to (1.18). Since the function $v_{\lambda}(x)=v(\lambda x), \lambda>0$, also solves (1.18), we conclude that $v_{\lambda}$ satisfies (1.19), but this implies

$$
\|\nabla v\|_{L^{\infty}\left(\mathbb{R}^{N}\right)} \leq \frac{1}{\lambda}\left(C_{1}+\frac{C_{2}}{\lambda^{\alpha(N-2) / 2}}\|\nabla v\|_{L^{2}\left(\mathbb{R}^{N}\right)}^{\alpha}\right) .
$$

Then, letting $\lambda \rightarrow \infty$, we deduce that $v$ is constant. Therefore an estimate such as (1.19) does not hold for (1.18) and probably neither for $\left(\mathrm{TW}_{c}\right)$. This a big difference with the semilinear equation (1.5). Indeed, if $\Psi$ is a solution of (1.5), then (see [11, 2])

$$
\|\Psi\|_{C^{k}\left(\mathbb{R}^{N}\right)} \leq C(c, k, N) .
$$

In dimension $N=2$, Hélein [22, 23] proved that any finite energy solution of (1.18) is continuous and therefore smooth. In dimension $N \geq 3$, this result if false. In fact, if $N \geq 3, v(x)=x /|x|$ is a discontinuous finite energy solution and Rivière [43] proved that (1.18) has almost everywhere discontinuous solutions with finite energy.

For these reasons, we need to treat differently the cases $N=2$ and $N \geq 3$. In any case, we establish in Section 2 a bound of $\|\nabla u\|_{L^{\infty}\left(\mathbb{R}^{N}\right)}$ in terms of the energy, provided that the energy is small enough.

Proposition 1.4. Let $c \geq 0$ and $u \in \mathcal{E}\left(\mathbb{R}^{2}\right)$ be a solution of $\left(\mathrm{TW}_{c}\right)$. Then $u \in C^{\infty}\left(\mathbb{R}^{2}\right), u_{3} \in L^{p}\left(\mathbb{R}^{2}\right)$ for all $p \in[2, \infty]$ and $\nabla u \in W^{k, p}\left(\mathbb{R}^{2}\right)$ for all $k \in \mathbb{N}$ and $p \in[2, \infty]$. Moreover, there exist constants $\varepsilon_{0}>0$ and $K>0$, independent of $u$ and $c$, such that

$$
\begin{aligned}
\left\|u_{3}\right\|_{L^{\infty}\left(\mathbb{R}^{2}\right)} & \leq K(1+c) E(u)^{1 / 2}, \\
\|\nabla u\|_{L^{\infty}\left(\mathbb{R}^{2}\right)} & \leq K(1+c) E(u)^{1 / 4},
\end{aligned}
$$

provided that $E(u) \leq \varepsilon_{0}$.

Denoting by $U C\left(\mathbb{R}^{N}\right)$ the set of uniformly continuous functions, in the higher dimensional case, we have

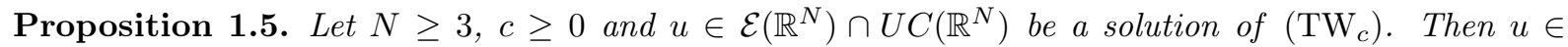
$C^{\infty}\left(\mathbb{R}^{N}\right)$ and $\nabla u \in W^{k, p}\left(\mathbb{R}^{N}\right)$ for all $k \in \mathbb{N}$ and $p \in[2, \infty]$. Moreover, if $N \in\{3,4\}$ and $c \in[0,1]$, there exist $\varepsilon_{0}, K, \alpha>0$, independent of $u$ and $c$, such that

$$
\begin{gathered}
\left\|u_{3}\right\|_{L^{\infty}\left(\mathbb{R}^{N}\right)} \leq K E(u)^{\alpha}, \\
\|\nabla u\|_{L^{\infty}\left(\mathbb{R}^{N}\right)} \leq K E(u)^{\alpha},
\end{gathered}
$$

provided that $E(u) \leq \varepsilon_{0}$.

As we will show, Propositions 1.4 and 1.5 will be enough to get the universal constant $M$ in estimate (E3). The proof of Proposition 1.5] is the only point of the paper where the condition $N \leq 4$ is used. It is straightforward to verify that if the estimates (1.22) and (1.23) are satisfy for some dimension $N$, then Theorem 1.1 holds for this $N$.

\subsubsection{Estimates (E1) and (E2)}

In order to prove Theorem 1.1, we can assume that $E(u)$ is small. Then, by (1.20) and (1.22), we only need to prove (E1) and (E2) for traveling waves such that $\left\|u_{3}\right\| \leq 1 / 2$.

In Section 3, we will prove that estimate (E1) holds with $p=4, \gamma=1$ if $N=2, p=2, \gamma=\frac{2 N+3}{2(N-1)}$ if $N \geq 3$, and $k=1, q=\infty$ in both cases. The main element in the proof is the study of the Fourier multiplier $\mathcal{L}_{c}$ done by the author in [9] if $N \geq 3$ and by Chiron and Maris [7] if $N=2$.

Sections 4 and 5 are devoted to establish some Pohozaev identities and a priori bounds that allow us to obtain estimate (E2). More precisely, under the condition $\left\|u_{3}\right\| \leq 1 / 2$, we show that

$$
E(u) \leq K\left\|u_{3}\right\|_{L^{p}}^{\delta},
$$

with $p=4, \delta=4$ if $N=2$, and $p=2, \delta=2$ if $N \geq 3$. 


\subsection{Asymptotic behavior at infinity}

As remarked before, the arguments given by Gravejat in [14, 16] apply to (1.15) and (1.17), since they rely mainly on the structure of the kernels. This allows to establish the precise limit at infinity of the finite energy solutions of $\left(\mathrm{TW}_{c}\right)$.

Theorem 1.6. Let $N \geq 2$ and $c \in(0,1)$. Assume that $u \in \mathcal{E}\left(\mathbb{R}^{N}\right)$ is a solution of $\left(\mathrm{TW}_{c}\right.$ ). Suppose further that $u \in U C\left(\mathbb{R}^{N}\right)$ if $N \geq 3$. Then there exist a constant $\lambda_{\infty} \in \mathbb{C}$ of modulus one and two functions $\check{u}_{\infty}, u_{3, \infty} \in C\left(\mathbb{S}^{N-1} ; \mathbb{R}\right)$ such that

$$
\begin{aligned}
|x|^{N-1}\left(\check{u}(x)-\lambda_{\infty}\right)-i \lambda_{\infty} \check{u}_{\infty}\left(\frac{x}{|x|}\right) & \rightarrow 0, \\
|x|^{N} u_{3}(x)-u_{3, \infty}\left(\frac{x}{|x|}\right) & \rightarrow 0,
\end{aligned}
$$

uniformly as $|x| \rightarrow \infty$. Moreover, assuming without loss of generality that $\lambda_{\infty}=1$, we have

$$
\begin{aligned}
\check{u}_{\infty}(\sigma) & =\frac{\alpha \sigma_{1}}{\left(1-c^{2}+c^{2} \sigma_{1}^{2}\right)^{\frac{N}{2}}}+\sum_{j=2}^{N} \frac{\beta_{j} \sigma_{j}}{\left(1-c^{2}+c^{2} \sigma_{1}^{2}\right)^{\frac{N}{2}}}, \\
u_{3, \infty}(\sigma) & =\alpha c\left(\frac{1}{\left(1-c^{2}+c^{2} \sigma_{1}^{2}\right)^{\frac{N}{2}}}-\frac{N \sigma_{1}^{2}}{\left(1-c^{2}+c^{2} \sigma_{1}^{2}\right)^{\frac{N+2}{2}}}\right)-\sum_{j=2}^{N} \beta_{j} \frac{N c \sigma_{1} \sigma_{j}}{\left(1-c^{2}+c^{2} \sigma_{1}^{2}\right)^{\frac{N+2}{2}}},
\end{aligned}
$$

where $\sigma=\left(\sigma_{1}, \ldots, \sigma_{N}\right) \in \mathbb{S}^{N-1}$,

$$
\alpha=\frac{\Gamma\left(\frac{N}{2}\right)}{2 \pi^{\frac{N}{2}}}\left(1-c^{2}\right)^{\frac{N-3}{2}}\left(2 c \int_{\mathbb{R}^{N}} e(u) u_{3} d x-\left(1-c^{2}\right) \int_{\mathbb{R}^{N}} G_{1}(x) d x\right)
$$

and

$$
\beta_{j}=-\frac{\Gamma\left(\frac{N}{2}\right)}{2 \pi^{\frac{N}{2}}}\left(1-c^{2}\right)^{\frac{N-1}{2}} \int_{\mathbb{R}^{N}} G_{j}(x) d x
$$

In particular, since the solutions found in [33] are uniformly continuous, Theorem 1.6 applies to those solutions. For the sake of completeness we sketch the proof of Theorem 1.6 in Section 7.

Remark 1.7. The analogous constants $\alpha$ and $\beta_{j}$ found in [16] for the asymptotic behavior at infinity of the nontrivial finite energy traveling waves to the Gross-Pitaevskii equation (1.5) can be written in terms the energy and (vectorial) momentum. Later, Wei and Yao [47] have proved that all the coordinates of the momentum of these solutions are zero, except the first one. As a consequence the traveling waves are asymptotically axisymmetric (see [47] for details). It is not clear if the arguments in [4] can be generalized in the context of the Landau-Lifshitz equation. However, we think that the same conclusion holds in our case, that is $\beta_{j}=0$, for all $2 \leq j \leq N$, which would imply the asymptotically axisymmetry of the solutions of $\left(\mathrm{TW}_{c}\right)$.

Notations. We use the standard notations "." and " $\times$ " for the inner and cross product, respectively.

For $y \in \mathbb{R}^{N}$ and $r \geq 0, B(y, r)$ or $B_{r}(y)$ denote the open ball of center $y$ and radius $r$ (which is empty for $r=0)$. In the case that there is no confusion, we simply put $B_{r}$.

Given $x=\left(x_{1}, x_{2}\right), f: \mathbb{R}^{2} \rightarrow \mathbb{R}^{2}, f=\left(f_{1}, f_{2}\right)$, we set $x^{\perp}=\left(-x_{2}, x_{1}\right)$, and $\operatorname{curl}(f)=\partial_{1} f_{2}-\partial_{2} f_{1}$. We also use the skew gradient $\nabla^{\perp}=\left(-\partial_{2}, \partial_{1}\right)$.

For a function $g: \mathbb{R}^{\ell} \rightarrow \mathbb{R}^{3}, g=\left(g_{1}, g_{2}, g_{3}\right)$, we define $\check{g}$ as the complex-valued function $\check{g}=g_{1}+i g_{2}$. We identify $\nabla g$ with the matrix in $\mathbb{R}^{\ell, 3}$ whose columns are $\nabla g_{1}, \nabla g_{2}$ and $\nabla g_{3}$.

Let $A=\left[A_{1}\left|A_{2}\right| A_{3}\right]$ and $\tilde{A}=\left[\tilde{A}_{1}\left|\tilde{A}_{2}\right| \tilde{A}_{3}\right]$ be two matrices in $\mathbb{R}^{\ell, 3}$, then

$$
A: \tilde{A}=\sum_{j=1}^{3} A_{j} \cdot \tilde{A}_{j},
$$

and for any vector $b \in \mathbb{R}^{3}, A . b \in \mathbb{R}^{3}$. denotes the standard matrix-vector product. 
Either $\mathcal{F}(f)$ or $\widehat{f}$ stands for the Fourier transform of $f$, namely

$$
\mathcal{F}(f)(\xi)=\widehat{f}(\xi)=\int_{\mathbb{R}^{N}} f(x) e^{-i x \cdot \xi} d x
$$

We also adopt the standard notation $K(\cdot, \cdot, \ldots)$ to represent a generic constant that depends only on each of its arguments.

\section{Estimates for $\left\|u_{3}\right\|_{L^{\infty}\left(\mathbb{R}^{N}\right)}$ and $\|\nabla u\|_{L^{\infty}\left(\mathbb{R}^{N}\right)}$}

In this section we use some of the elements developed to the study of the harmonic map equation. In particular, the next lemma is a consequence of the Wente lemma [48, 6, 46] and Hélein's trick [22, 23.

Lemma 2.1. Let $\Omega \subset \mathbb{R}^{2}$ be a smooth bounded domain and $g \in L^{2}(\Omega)$. Assume that $u \in H^{1}\left(\Omega, \mathbb{S}^{2}\right)$ satisfies

$$
-\Delta u=|\nabla u|^{2} u+g, \quad \text { in } \Omega .
$$

Let $r>0$ and $x \in \Omega$ such that $B(x, r) \subseteq \Omega$. Then for any $i \in\{1,2,3\}$ we have

$$
\begin{aligned}
\underset{B(x, r / 2)}{\mathrm{osc}} u_{i} \leq & K\left(\min \left\{\|\nabla u\|_{L^{2}(B(x, r))},\left\|u_{i}\right\|_{L^{\infty}\left(\partial B_{r}\right)}\right\}+\|\nabla u\|_{L^{2}(B(x, r))}^{2}\right. \\
& \left.+r\|g\|_{L^{2}(B(x, r))}\left(1+\|\nabla u\|_{L^{2}(B(x, r))}\right)\right)
\end{aligned}
$$

for some universal constant $K>0$. In particular $u \in C(\Omega)$. Moreover, if the trace of $u$ on $\partial \Omega$ belongs to $C(\partial \Omega)$, then $u \in C(\bar{\Omega})$ and

$$
\left\|u_{i}\right\|_{L^{\infty}(\Omega)} \leq\left\|u_{i}\right\|_{L^{\infty}(\partial \Omega)}+K(\Omega)\left(\|\nabla u\|_{L^{2}(\Omega)}^{2}+\|g\|_{L^{2}(\Omega)}\left(1+\|\nabla u\|_{L^{2}(\Omega)}\right)\right),
$$

for some constant $K(\Omega)$ depending only on $\Omega$.

Proof. As for the standard harmonic maps, we recast (2.1) as

$$
-\Delta u_{i}=\sum_{j=1}^{3} v_{i, j} \cdot \nabla u_{j}+g_{i}, \quad \text { in } \Omega, \quad i=1,2,3,
$$

where $v_{i, j}=u_{i} \nabla u_{j}-u_{j} \nabla u_{i}$. Then

$$
\operatorname{div}\left(v_{i, j}\right)=u_{j} g_{i}-u_{i} g_{j} \quad \text { and } \quad\left\|\operatorname{div}\left(v_{i, j}\right)\right\|_{L^{2}(\Omega)} \leq 2\|g\|_{L^{2}(\Omega)} .
$$

Let us consider $h_{i, j} \in H^{2}(\Omega)$ the solution of

$$
\begin{cases}\Delta h_{i, j}=\operatorname{div}\left(v_{i, j}\right), & \text { in } \Omega \\ h_{i, j}=0, & \text { on } \partial \Omega\end{cases}
$$

Thus

$$
\left\|\nabla h_{i, j}\right\|_{L^{2}(\Omega)} \leq\left\|v_{i, j}\right\|_{L^{2}(\Omega)} \leq 2\|\nabla u\|_{L^{2}(\Omega)} .
$$

Since $\operatorname{div}\left(v_{i, j}-\nabla h_{i, j}\right)=0$, with $v_{i, j}-\nabla h_{i, j} \in L^{2}(\Omega)$, there exists $w_{i, j} \in H^{1}(\Omega)$ (see e.g. [13, Thm 2.9]) such that

$$
v_{i, j}=\nabla h_{i, j}+\nabla^{\perp} w_{i, j}, \quad \text { in } \Omega .
$$

Now we decompose $u$ as $u_{i}=\phi_{i}+\varphi_{i}+\psi_{i}$, where $\phi_{i}, \varphi_{i}, \psi_{i}$ are the solutions of the equations

$$
\begin{gathered}
\begin{cases}-\Delta \phi_{i}=0, & \text { in } U, \\
\phi_{i}=u_{i}, & \text { on } \partial U,\end{cases} \\
\begin{cases}-\Delta \varphi_{i}=\nabla h_{i}: \nabla u+g_{i}, & \text { in } U, \\
\varphi_{i}=0, & \text { on } \partial U,\end{cases}
\end{gathered}
$$




$$
\begin{cases}-\Delta \psi_{i}=\nabla^{\perp} w_{i}: \nabla u, & \text { in } U, \\ \psi_{i}=0, & \text { on } \partial U,\end{cases}
$$

where $\nabla h_{i}=\left[\nabla h_{i, 1}\left|\nabla h_{i, 2}\right| \nabla h_{i, 3}\right], \nabla^{\perp} w_{i}=\left[\nabla^{\perp} w_{i, 1}\left|\nabla^{\perp} w_{i, 2}\right| \nabla^{\perp} w_{i, 3}\right]$, and $U$ is an open smooth domain such that $B_{r} \subseteq U \subseteq \Omega$. We now prove (2.2) for $r=1$, supposing that $B_{1} \subseteq U$, since then (2.2) follows from a scaling argument. First, invoking Theorem A.1 we have that

$$
\underset{B_{1 / 2}}{\operatorname{osc}} \phi_{i} \leq K \min \left\{\left\|\nabla \phi_{i}\right\|_{L^{2}\left(B_{2 / 3}\right)},\left\|\phi_{i}\right\|_{L^{2}\left(B_{2 / 3}\right)}\right\} .
$$

Also, some standard computations and the maximum principle yield

$$
\left\|\nabla \phi_{i}\right\|_{L^{2}(U)} \leq\left\|\nabla u_{i}\right\|_{L^{2}(U)} \quad \text { and } \quad\left\|\phi_{i}\right\|_{L^{\infty}(U)} \leq\left\|u_{i}\right\|_{L^{\infty}(\partial U)} .
$$

Thus from (2.12) and (2.13) we conclude that

$$
\underset{B_{1 / 2}}{\operatorname{osc}} \phi_{i} \leq K \min \left\{\left\|\nabla u_{i}\right\|_{L^{2}(U)},\left\|u_{i}\right\|_{L^{\infty}(\partial U)}\right\} .
$$

For $\varphi_{i}$, Theorem $\mathrm{A} .2$ gives

$$
\underset{B_{1}}{\operatorname{Osc}} \varphi_{i} \leq K\left(\|\nabla h \cdot \nabla u\|_{L^{3 / 2}\left(B_{1}\right)}+\|g\|_{L^{2}\left(B_{1}\right)}\right) .
$$

To estimate the first term in the r.h.s. of (2.15), we use the Hölder inequality

$$
\left\|\nabla h_{i}: \nabla u\right\|_{L^{3 / 2}\left(B_{1}\right)} \leq\|\nabla h\|_{L^{6}\left(B_{1}\right)}\|\nabla u\|_{L^{2}\left(B_{1}\right)},
$$

and the Sobolev embedding theorem

$$
\|\nabla h\|_{L^{6}\left(B_{1}\right)} \leq K\left(\|\nabla h\|_{L^{2}\left(B_{1}\right)}+\left\|D^{2} h\right\|_{L^{2}\left(B_{1}\right)}\right) .
$$

By using (2.5), (2.15), (2.16), (2.17) and $L^{2}$-regularity estimates for (2.6), we are led to

$$
\underset{B_{1}}{\operatorname{Osc}} \varphi_{i} \leq K\|g\|_{L^{2}\left(B_{1}\right)}\left(1+\|\nabla u\|_{L^{2}\left(B_{1}\right)}\right) .
$$

Similarly, since $W^{2, p}(U) \hookrightarrow C(\bar{U})$, for all $p>1$, we also have

$$
\left\|\varphi_{i}\right\|_{C(\bar{U})} \leq K(U)\|g\|_{L^{2}(U)}\left(1+\|\nabla u\|_{\left.L^{2}(U)\right)}\right) .
$$

To estimate $\psi_{i}$ we invoke the Wente estimate (see [46], [23]), so that

$$
\left\|\psi_{i}\right\|_{C(\bar{U})}+\underset{U}{\operatorname{osc}} \psi_{i} \leq K\|\nabla w\|_{L^{2}(U)}\|\nabla u\|_{L^{2}(U)} \leq K\|\nabla u\|_{L^{2}(U)}^{2},
$$

where we have used (2.7) and (2.8) for the last inequality.

Therefore, taking $U=B_{1}$ and putting together (2.14), (2.18) and (2.20), we conclude (2.2) with $r=1$.

If the trace of $u$ on $\partial \Omega$ belongs to $C(\partial \Omega)$, we take $\Omega=U$ and then from (2.9) we deduce that $\phi_{i} \in C^{2}(\Omega) \cap C(\bar{\Omega})$. Since $\varphi_{i}, \psi_{i} \in C(\bar{\Omega})$, we conclude that $u_{i} \in C(\bar{\Omega})$ and (2.3) follows from (2.13), (2.19) and (2.20).

Lemma 2.2. Let $y \in \mathbb{R}^{2}, r>0$ and $B_{r} \equiv B(y, r)$. Assume that $u \in H^{1}\left(B_{r}, \mathbb{S}^{2}\right)$ satisfies

$$
-\Delta u=|\nabla u|^{2} u+f(x, u(x), \nabla u(x)), \quad \text { in } B_{r},
$$

where $f$ is a continuous function such that $|f(x, z, p)| \leq C_{1}+C_{2}|p|$, for some constants $C_{1}, C_{2} \geq 0$, for a.e. $x \in B_{r}, z \in \mathbb{R}^{3}, p \in \mathbb{R}^{3 \times 3}$. Suppose that

$$
A \equiv A(u, r) \equiv \frac{\operatorname{osc}_{B_{r}} u\left(1+r^{2}\left(C_{1}+C_{2}^{2}\right)\right)}{1-3 \operatorname{osc}_{B_{r}} u} \leq \frac{1}{32} .
$$

Then

$$
\left\|D^{2} u\right\|_{L^{2}\left(B_{r / 2}\right)}+\|\nabla u\|_{L^{4}\left(B_{r / 2}\right)}^{2} \leq K r^{-1}\left(\|\nabla u\|_{L^{2}\left(B_{r}\right)}+\|g\|_{L^{2}\left(B_{r}\right)}\right),
$$


where $g(x)=f(x, u(x), \nabla u(x))$. Assume further that $f(x, z, p)=\tilde{f}(x)+R_{f}(x, z, p)$, for some continuous functions $\tilde{f}, R_{f}$, such that $\left|R_{f}(x, z, p)\right| \leq C_{3}|p|$, for some constant $C_{3} \geq 0$, for a.e. $x \in B_{r}, z \in \mathbb{R}^{3}$, $p \in \mathbb{R}^{3 \times 3}$. Then,

$$
\begin{aligned}
\|\nabla u\|_{L^{\infty}\left(B_{r / 4}\right)} \leq & K r^{-1}\|\nabla u\|_{L^{2}\left(B_{r}\right)}+K r^{-2 / 3}\left(\|\nabla u\|_{L^{2}\left(B_{r}\right)}^{2}\left(r^{-2}+r^{-4 / 3}\right)+\|g\|_{L^{2}\left(B_{r}\right)}^{2}\right. \\
& \left.+\|\tilde{f}\|_{L^{3}\left(B_{r}\right)}+C_{3} r^{-1 / 3}\|\nabla u\|_{L^{2}\left(B_{r}\right)}^{1 / 3}\left(\|\nabla u\|_{L^{2}\left(B_{r}\right)}^{1 / 3}+\|g\|_{L^{2}\left(B_{r}\right)}^{1 / 3}\right)\right)
\end{aligned}
$$

where $K$ is some universal constant.

Proof. As mentioned before, Lemma 2.1 and the quadratic growth of the r.h.s. of (2.21) imply that $u \in H_{\text {loc }}^{2,2}(\Omega)$. In fact, this could be seen by repeating the following arguments with finite differences instead of weak derivatives. As standard in the analysis of this type of equations, we let $\rho \in(0, r)$ and $\chi \in C_{0}^{\infty}\left(B_{r}\right)$, with $\chi(x)=1$ if $|x| \leq \rho$,

$$
|\chi| \leq 1 \quad \text { and } \quad|\nabla \chi| \leq K /(r-\rho), \quad \text { on } B_{r} .
$$

Then setting $\eta=\chi|\nabla u|$, taking inner product in (2.1) with $\left(u-u\left(x_{0}\right)\right) \eta^{2}$ and integrating by parts we obtain

$$
\int_{B_{r}}|\nabla u|^{2} \eta^{2}+2 \int_{B_{r}}\left(\nabla u \cdot\left(u-u\left(x_{0}\right)\right)\right) \cdot(\eta \nabla \eta)=\int_{B_{r}}|\nabla u|^{2} u \cdot\left(u-u\left(x_{0}\right)\right) \eta^{2}+\int_{B_{r}} \eta^{2} g \cdot\left(u-u\left(x_{0}\right)\right) .
$$

Then, using the elementary inequality $2 a b \leq a^{2}+b^{2}$,

$$
\begin{gathered}
\left|\eta^{2} g \cdot\left(u-u\left(x_{0}\right)\right)\right| \leq \eta^{2}\left(C_{1}+C_{2}|\nabla u|\right) \underset{B_{r}}{\operatorname{osc}}(u) \leq C_{1} \eta^{2} \underset{B_{r}}{\operatorname{osc}}(u)+ \\
\eta^{2}|\nabla u|^{2} \underset{B_{r}}{\operatorname{osc}}(u)+\frac{1}{4} C_{2}^{2} \eta^{2} \underset{B_{r}}{\operatorname{osc}}(u) .
\end{gathered}
$$

In a similar fashion, we estimate the remaining terms in (2.26). Then, using the Poincaré inequality

$$
\|\eta\|_{L^{2}\left(B_{r}\right)} \leq \frac{r}{j_{0}}\|\nabla \eta\|_{L^{2}\left(B_{r}\right)}
$$

where $j_{0} \approx 2.4048$ is the first zero of the Bessel function, and that $|u|=1$, we conclude that

$$
\int_{B_{r}}|\nabla u|^{2} \eta^{2} \leq \frac{\operatorname{osc}_{B_{r}} u\left(1+r^{2}\left(C_{1}+C_{2}^{2}\right)\right)}{1-3 \operatorname{osc}_{B_{r}} u} \int_{B_{r}}|\nabla \eta|^{2},
$$

where we bounded $1 / j_{0}$ and $1 /\left(4 j_{0}\right)$ by 1 to simplify the estimate. Thus,

$$
\left.\int_{B_{r}}|\nabla u|^{4} \chi^{2} \leq A \int_{B_{r}}\left(\left|D^{2} u\right|^{2} \chi^{2}+|\nabla u|^{2}|\nabla \chi|^{2}\right)\right) .
$$

On the other hand, taking inner product in (2.21) with $\partial_{k}\left(\chi^{2} \partial_{k} u\right)$, integrating by parts and summing over $k=1,2$, we have

$$
-\int_{B_{r}} \chi^{2}\left|D^{2} u\right|^{2}-2 \sum_{\substack{i \in\{1,2\} \\ j, k \in\{1,2,3\}}} \int_{B_{r}} \partial_{j k} u_{i} \chi \partial_{j} \chi \partial_{k} u_{i}=\sum_{i \in\{1,2\}} \int_{B_{r}}\left(|\nabla u|^{2} u_{i}+g_{i}\right)\left(2 \chi \nabla \chi \nabla u_{i}+\chi^{2} \Delta u_{i}\right) .
$$

Using again the inequalities $2 a b \leq \varepsilon a^{2}+b^{2} / \varepsilon$ and $a b \leq \varepsilon a^{2}+b^{2} / 4 \varepsilon$, we are led to

$$
\left.\int_{B_{r}} \chi^{2}\left|D^{2} u\right|^{2} \leq \frac{1}{1-3 \varepsilon} \int_{B_{r}}\left(\left(2+\varepsilon^{-1}\right)|\nabla u|^{2}|\nabla \chi|^{2}+\left(1+4 \varepsilon^{-1}\right)|\nabla u|^{4} \chi^{2}+\left(1+4 \varepsilon^{-1}\right) \chi^{2}\left|g^{2}\right|\right)\right) .
$$

Then, minimizing with respect to $\varepsilon$, it follows that

$$
\int_{B_{r}} \chi^{2}\left|D^{2} u\right|^{2} \leq 16 \int_{B_{r}}\left(|\nabla u|^{2}|\nabla \chi|^{2}+|\nabla u|^{4} \chi^{2}+\chi^{2}\left|g^{2}\right|\right) .
$$


By combining (2.25), (2.27) and (2.30), we infer that

$$
\begin{gathered}
\int_{B_{\rho}}|\nabla u|^{4} \leq \frac{K A}{1-16 A}\left(\frac{1}{(r-\rho)^{2}} \int_{B_{r}}|\nabla u|^{2}+\int_{B_{r}}|g|^{2}\right), \\
\int_{B_{\rho}}\left|D^{2} u\right|^{2} \leq \frac{K}{1-16 A}\left(\frac{1+A}{(r-\rho)^{2}} \int_{B_{r}}|\nabla u|^{2}+\int_{B_{r}}|g|^{2}\right) .
\end{gathered}
$$

Taking $\rho=r / 2$ and using that $A \leq 1 / 32$, (2.23) follows.

Now we decompose $u$ as $u_{i}=\phi_{i}+\psi_{i}$, where

$$
\begin{gathered}
\begin{cases}-\Delta \phi_{i}=0, & \text { in } B_{r / 2}, \\
\phi_{i}=u_{i}, & \text { on } \partial B_{r / 2},\end{cases} \\
\begin{cases}-\Delta \psi_{i}=|\nabla u|^{2} u_{i}+\tilde{f}_{i}+\left(R_{f}(x, u, \nabla u)\right)_{i}, & \text { in } B_{r / 2}, \\
\psi_{i}=0, & \text { on } \partial B_{r / 2},\end{cases}
\end{gathered}
$$

Since $\phi_{i}$ is a harmonic function,

$$
\left\|\nabla \phi_{i}\right\|_{L^{\infty}\left(B_{r / 4}\right)} \leq K r^{-1}\left\|\nabla \phi_{i}\right\|_{L^{2}\left(B_{r / 2}\right)},
$$

so that using also (2.13), we obtain the estimate

$$
\left\|\nabla \phi_{i}\right\|_{L^{\infty}\left(B_{r / 4}\right)} \leq K r^{-1}\left\|\nabla u_{i}\right\|_{L^{2}\left(B_{r / 2}\right)} .
$$

For $\psi_{i}$, we recall that using the $L^{p}$-regularity theory for the Laplacian and a scaling argument, the solution $v \in H_{0}^{1}\left(B_{R}\right)$ of the equation $-\Delta v=h$ satisfies

$$
\|\nabla v\|_{L^{\infty}\left(B_{R}\right)} \leq K(p) R^{1-2 / p}\|h\|_{L^{p}\left(B_{R}\right)}, \quad \text { for all } p>2 .
$$

Applying this estimate with $p=3$ to (2.34), we get

$$
\left\|\nabla \psi_{i}\right\|_{L^{\infty}\left(B_{r / 2}\right)} \leq C r^{-2 / 3}\left(\|\nabla u\|_{L^{6}\left(B_{r / 2}\right)}^{2}+\|\tilde{f}\|_{L^{3}\left(B_{r / 2}\right)}+C_{3}\|\nabla u\|_{L^{3}\left(B_{r / 2}\right)}\right) .
$$

Also, by the Sobolev embedding theorem, we have

$$
\|\nabla u\|_{L^{6}\left(B_{r / 2}\right)} \leq K\left(\left\|D^{2} u\right\|_{L^{2}\left(B_{r / 2}\right)}+r^{-2 / 3}\|\nabla u\|_{L^{2}\left(B_{r / 2}\right)}\right) .
$$

Therefore, by putting together (2.23), (2.35), (2.36), (2.37) and the interpolation inequality

$$
\|\nabla u\|_{L^{3}\left(B_{r / 2}\right)} \leq\|\nabla u\|_{L^{2}\left(B_{r / 2}\right)}^{1 / 3}\|\nabla u\|_{L^{4}\left(B_{r / 2}\right)}^{2 / 3},
$$

we deduce (2.24).

Now we turn back to equation $\left(\mathrm{TW}_{c}\right)$. By setting

$$
E_{x, r}(u)=\int_{B(x, r)} e(u),
$$

we obtain the following result.

Corollary 2.3. There exist $\varepsilon_{0}>0$ and a positive constant $K\left(\varepsilon_{0}\right)$, such that for any $c \geq 0$ and any $u \in \mathcal{E}\left(\mathbb{R}^{2}\right)$ solution of $\left(\mathrm{TW}_{c}\right)$ satisfying

$$
E_{x, r}(u) \leq \varepsilon_{0}
$$

for some $x \in \mathbb{R}^{2}$ and $r \in(0,1]$, we have

$$
\begin{aligned}
\underset{B(x, r / 2)}{\operatorname{Osc}} u & \leq K\left(\varepsilon_{0}\right)(1+c) E_{x, r}(u)^{1 / 2}, \\
\|\nabla u\|_{L^{\infty}(B(x, r / 4))} & \leq K\left(\varepsilon_{0}\right)(1+c) E_{x, r}(u)^{1 / 4} r^{-2 / 3} .
\end{aligned}
$$

In particular, if $E(u) \leq \varepsilon_{0}$, then (1.20) and (1.21) hold. 
Proof. Estimates (2.38) and (2.39) follow from Lemmas 2.1 and 2.2 Then, taking $r=1$, we conclude that (1.21) holds. Now we turn to (1.20). For any $y \in \mathbb{R}^{2}$ we have

$$
2 E(u) \geq \int_{B(y, 1 / 2)} u_{3}^{2} \geq \frac{\pi}{4} \min _{B(y, 1 / 2)}\left|u_{3}\right|^{2} .
$$

On the other hand, by Lemma 2.1

$$
\max _{B(y, 1 / 2)}\left|u_{3}\right| \leq \underset{B(y, 1 / 2)}{\operatorname{osc}} u_{3}+\min _{B(y, 1 / 2)}\left|u_{3}\right| \leq K(1+c) E(u)^{1 / 2}+\min _{B(y, 1 / 2)}\left|u_{3}\right| .
$$

By combining (2.40) and (2.41), we are led to (1.20).

Proof of Proposition 1.4. Since $u$ has finite energy, for every $\varepsilon>0$, there exists $\rho>0$ such that for all $y \in \mathbb{R}^{2}$

$$
E_{y, \rho}(u) \leq \varepsilon .
$$

In fact, since $e(u) \in L^{1}\left(\mathbb{R}^{2}\right)$, by Lemma A.3 for every $\varepsilon>0$ we can decompose $e(u)=e_{1, \varepsilon}(u)+e_{2, \varepsilon}(u)$ such that

$$
\left\|e_{1, \varepsilon}(u)\right\|_{L^{1}\left(\mathbb{R}^{2}\right)} \leq \varepsilon / 2 \quad \text { and } \quad\left\|e_{2, \varepsilon}(u)\right\|_{L^{\infty}\left(\mathbb{R}^{2}\right)} \leq K_{\varepsilon},
$$

for some constant $K_{\varepsilon}$. Then for any $y \in \mathbb{R}^{2}$,

$$
\left\|e_{2, \varepsilon}(u)\right\|_{L^{1}(B(y, \rho))} \leq K_{\varepsilon} \pi \rho^{2} .
$$

Taking

$$
\rho=\left(\frac{\varepsilon}{2 K_{\varepsilon} \pi}\right)^{1 / 2},
$$

we obtain (2.42). Thus, invoking Corollary 2.3. with $\varepsilon=\varepsilon_{0}$ and $r=\min \{1, \rho\}$, we conclude that

$$
\|\nabla u\|_{L^{\infty}(B(y, r / 4))} \leq K\left(\varepsilon_{0}\right)(1+c) E(u)^{1 / 4}, \quad \text { for all } y \in \mathbb{R}^{2} .
$$

Therefore $u \in W^{1, \infty}\left(\mathbb{R}^{2}\right)$, with $\|\nabla u\|_{L^{\infty}\left(\mathbb{R}^{2}\right)} \leq K\left(\varepsilon_{0}\right)(1+c) E(u)^{1 / 4}$. Differentiating $\left(\overline{T W_{c}}\right)$, we find that $v=\partial_{j} u, j=1,2$, satisfies

$$
L_{\lambda}(v) \equiv-\Delta v-2(\nabla u: \nabla v) u-c\left(u \times \partial_{1} v\right)+\lambda v=|\nabla u|^{2} v+2 u_{3} v_{3} u+u_{3}^{2} v-v_{3} e_{3}+c\left(v \times \partial_{1} u\right)+\lambda v,
$$

in $\mathbb{R}^{2}$. Since $\nabla u \in L^{\infty}\left(\mathbb{R}^{2}\right) \cap L^{2}\left(\mathbb{R}^{2}\right)$, we deduce that the r.h.s. of the formula above belongs to $L^{2}\left(\mathbb{R}^{2}\right)$. Therefore taking $\lambda>0$ large enough, we can invoke the elliptic regularity theory for linear systems and deduce that $v \in W^{2,2}\left(\mathbb{R}^{2}\right)$. Then, by the Sobolev embedding theorem, $D^{2} u \in L^{p}\left(\mathbb{R}^{2}\right)$, for all $p \in[2, \infty)$ and a bootstrap argument allows us to conclude that $\nabla u \in W^{k, p}\left(\mathbb{R}^{2}\right)$ for all $k \in \mathbb{N}$ and $p \in[2, \infty]$.

The estimates (1.20) and (1.21) are given by Corollary 2.3 ,

Proposition 1.4 shows that $u_{3}$ is uniformly continuous, so that $u_{3}(x) \rightarrow 0$, as $|x| \rightarrow \infty$. In particular $u$ belongs to the space $\tilde{\mathcal{E}}\left(\mathbb{R}^{2}\right)$, where

$$
\tilde{\mathcal{E}}\left(\mathbb{R}^{N}\right)=\left\{v \in \mathcal{E}\left(\mathbb{R}^{N}\right): \exists R \geq 0 \text { s.t. }\left\|v_{3}\right\|_{\left.L^{\infty}(B(0, R))^{c}\right)}<1\right\} .
$$

In the case $N \geq 3$, we always suppose that $u \in \mathcal{E}\left(\mathbb{R}^{N}\right) \cap U C\left(\mathbb{R}^{N}\right)$ and then it is immediate that $u \in \tilde{\mathcal{E}}\left(\mathbb{R}^{N}\right)$. Now we recall a well-known result (see e.g. [34, Proposition 2.5]) that provides the existence of the lifting for any function in $\tilde{\mathcal{E}}\left(\mathbb{R}^{N}\right)$.

Lemma 2.4. Let $N \geq 2$ and $v \in \tilde{\mathcal{E}}\left(\mathbb{R}^{N}\right)$. Then there exists $R \geq 0$ such that $v$ admits the lifting

$$
\check{v}(x)=\varrho(x) e^{i \theta(x)}, \quad \text { on } B(0, R)^{c},
$$

where $\varrho=\sqrt{1-v_{3}^{2}}$ and $\theta$ is a real-valued function. Moreover, $\varrho, \theta \in H_{\mathrm{loc}}^{1}\left(B(0, R)^{c}\right)$ and $\nabla \varrho, \nabla \theta \in$ $\left.L^{2}\left(B(0, R)^{c}\right)\right)$.

Corollary 2.5. Let $c \geq 0$ and $u \in \mathcal{E}\left(\mathbb{R}^{2}\right)$ be a solution of $\left(\overline{T W_{c}}\right)$. Then there is $R \geq 0$ such that the lifting $\check{u}(x)=\varrho(x) e^{i \theta(x)}$ holds on $B(0, R)^{c}$ and satisfies $\nabla \varrho, \nabla \theta \in W^{k, p}\left(B(0, R)^{c}\right)$ for any $k \geq 2$ and $p \in[2, \infty]$. Moreover, there exists a constant $\varepsilon(c)>0$, depending only on $c$, such that if $E(u) \leq \varepsilon(c)$, then we can take $R=0$. 
Proof. By Proposition 1.4, $u \in \tilde{\mathcal{E}}\left(\mathbb{R}^{2}\right)$ and then Lemma 2.4 gives us the existence of the lifting, whose properties follow from Proposition 1.4 and the identity

$$
|\nabla \check{u}|^{2}=\varrho^{2}|\nabla \theta|^{2}+|\nabla \varrho|^{2}, \quad \text { on } B(0, R)^{c},
$$

noticing that $1-\left\|v_{3}\right\|_{L^{\infty}\left(B(0, R)^{c}\right)}^{2}=\inf \left\{\varrho(x)^{2}: x \in B(0, R)^{c}\right\}>0$. The last assertion is an immediate consequence of (1.20).

In the case $N \geq 3$, some regularity for the solutions of the equation (2.1) can be obtained considering that $u$ is a stationary solution in the sense introduced by R. Moser in 37 .

Definition 2.6. Let $\Omega \subset \mathbb{R}^{N}$ be a smooth bounded domain and $g \in L^{p}\left(\Omega ; \mathbb{R}^{3}\right)$. A solution $u \in H^{1}\left(\Omega ; \mathbb{S}^{2}\right)$ of (2.1) is called stationary if

$$
\operatorname{div}\left(|\nabla u|^{2} e_{j}-2 \nabla u . \partial_{j} u\right)=2 \partial_{j} u \cdot g, \quad \text { in } \Omega,
$$

for all $j \in\{1, \ldots, N\}$ in the distributional sense.

If we suppose that $u$ is a smooth solution of (2.1), then

$$
\operatorname{div}\left(|\nabla u|^{2} e_{j}-2 \nabla u . \partial_{j} u\right)=-2 \Delta u \cdot \partial_{j} u=2 g \cdot \partial_{j} u,
$$

so it is a stationary solution. However not every solution $u \in H^{1}\left(\Omega ; \mathbb{S}^{2}\right)$ of (2.1) satisfies (2.45). The advantage of stationary solutions is that they satisfy a monotonicity formula that allows to generalize some standard results for harmonic maps. However, when $g$ belongs only to $L^{2}(\Omega)$, the regularity estimates hold only for $N \leq 4$.

Lemma 2.7 ([37]). Let $N \leq 4$ and $y \in \mathbb{R}^{N}$. Assume that $u \in H^{1}\left(B(y, 1) ; \mathbb{S}^{2}\right) \cap W^{1,4}(B(y, 1))$ is a stationary solution of (2.1), with $\Omega=B(y, 1)$ and $g \in L^{2}(B(y, 1))$. Then there exist $K>0$ and $\varepsilon_{0}>0$, depending only on $N$, such that if

$$
\|\nabla u\|_{L^{2}(B(y, 1))}+\|g\|_{L^{2}(B(y, 1))}=\varepsilon \leq \varepsilon_{0},
$$

we have

$$
\|\nabla u\|_{L^{4}(B(y, 1 / 4))} \leq K \varepsilon^{\frac{1}{2}} .
$$

Applying this result to equation $\left(\mathrm{TW}_{c}\right)$, we are led to the following estimate.

Lemma 2.8. Let $N \leq 4$. There exist $K>0$ and $\varepsilon_{0}>0$, depending only on $N$, such that for any solution $u \in \mathcal{E}\left(\mathbb{R}^{N}\right) \cap C^{\infty}\left(\mathbb{R}^{N}\right)$ of $\left(\mathrm{TW}_{c}\right)$, with $c \in[0,1]$, satisfying $E(u) \leq \varepsilon_{0}$, we have

$$
\|\nabla u\|_{L^{4}(B(x, 1))} \leq K E(u)^{1 / 2} .
$$

Now we are in position to complete the regularity result in higher dimensions stated in the introduction.

Proof of Proposition 1.5. Recalling again a classical results for elliptic systems with quadratic growth (see [5, 31, 28]), $u \in U C\left(\mathbb{R}^{N}\right)$ yields that $u \in C^{\infty}\left(\mathbb{R}^{N}\right)$. This is due to the fact that now we are assuming that $u$ is uniformly continuous and then we can choose $r>0$ small such that the oscillation of $u$ on the ball $B(y, r)$ is small, uniformly in $y$. Then we can make the quantity $A(u, r)$ defined in (2.22) as small as needed and repeat the first part of the proof of Lemma 2.2 to conclude that for all $y \in \mathbb{R}^{N}$

$$
\left\|D^{2} u\right\|_{L^{2}(B(y, r / 2))}+\|\nabla u\|_{L^{4}(B(y, r / 2))}^{2} \leq K(N) r^{-1}\left(\|\nabla u\|_{L^{2}(B(y, r))}+\left\|u_{3}\right\|_{L^{2}(B(y, r))}\right),
$$

for some constant $K(N)$ and $r>0$ small enough, independent of $y$. At this stage we note that we cannot follow the rest of the argument of Lemma 2.2 since it relies on the two-dimensional Sobolev embeddings. However, it is well-known that using (2.46) it is possible to deduce that $\nabla u \in L^{p}\left(\mathbb{R}^{N}\right)$, for all $p \geq 2$. More precisely, as discussed before, there exists $r \in(0,1]$ such that

$$
\underset{B\left(y, 2^{N} r\right)}{\operatorname{osc}} u \leq \frac{1}{8(1+c)(2 N-1)}, \quad \text { for all } y \in \mathbb{R}^{N} .
$$


Then, by iterating Lemma A.5, we have

$$
\int_{B(y, r)}|\nabla u|^{2 N+2} \leq K(N)(1+c)^{2 N} \frac{E(u)}{r^{2 N}}, \quad \text { for all } y \in \mathbb{R}^{N} .
$$

By proceeding as in the proof of Lemma 2.2, we decompose $u_{i}$ as $u_{i}=\phi_{i}+\psi_{i}$, where

$$
\begin{gathered}
\begin{cases}-\Delta \phi_{i}=0, & \text { in } B(y, r), \\
\phi_{i}=u_{i}, & \text { on } \partial B(y, r),\end{cases} \\
\begin{cases}-\Delta \psi_{i}=|\nabla u|^{2} u_{i}+u_{3}^{2} u_{i}-\delta_{i, 3} u_{3}+c u \times \partial_{1} u, & \text { in } B(y, r), \\
\psi_{i}=0, & \text { on } \partial B(y, r) .\end{cases}
\end{gathered}
$$

In view of (2.48), elliptic regularity estimates imply that $\psi_{i} \in W^{2, N+1}(B(y, r))$ and then by the Sobolev embedding theorem we can establish an upper bound for $\left\|\nabla \psi_{i}\right\|_{L^{\infty}(B(y, r))}$ in terms of powers of $E(u)$. Since $\phi_{i}$ is a harmonic function, we obtain a similar estimate for $\phi_{i}$ as in the proof of Lemma 2.24. Then we conclude that $\nabla u \in L^{\infty}\left(\mathbb{R}^{N}\right)$, so that, by interpolation, $\nabla u \in L^{p}\left(\mathbb{R}^{N}\right)$, for all $p \in[2, \infty]$. Proceeding as in the proof of Proposition [1.4, we conclude that $\nabla u \in W^{k, p}\left(\mathbb{R}^{N}\right)$, for all $k \in \mathbb{N}$ and $p \in[2, \infty]$.

Now we turn to (1.22) and (1.23). Let us first take $N=3$ and $\varepsilon_{0}$ given by Lemma 2.8, such that $E(u) \leq \varepsilon_{0}$. Then, by the Morrey inequality,

$$
\underset{B(y, 1 / 2)}{\operatorname{Osc}} u \leq K\|\nabla u\|_{L^{4}(B(y, 1))} \leq K E(u)^{1 / 2}
$$

for all $y \in \mathbb{R}^{3}$ and for all $c \in[0,1]$. Taking $\varepsilon_{0}$ smaller if necessary, (2.47) holds with $r=1 / 16$ and then so it does (2.48) (with $r=1 / 16)$. Hence the previous computations give a bound for $\nabla u \in L^{\infty}\left(\mathbb{R}^{3}\right)$ depending only on $E(u)$, which yields (1.23).

In order to prove (1.22), we estimate the minimum of $\left|u_{3}\right|$ on $B(y, 1 / 2)$ as in (2.40), and using (2.49) we conclude that

which implies (1.22).

$$
\max _{B(y, 1 / 2)}\left|u_{3}\right| \leq K E(u)^{1 / 2}
$$

It only remains to consider the case $N=4$. Note that the r.h.s. of (2.47) is less than or equal to $1 / 112$, for $c \in[0,1]$. Let $r_{*}>0$ be the maximal radius given by the uniform continuity of $u$ for this value, i.e.

$$
r_{*}=\sup \left\{\rho>0: \forall x, z \in \mathbb{R}^{4},|x-z| \leq \rho \Rightarrow|u(x)-u(z)| \leq 1 / 112\right\} .
$$

We claim that $r_{*} \geq 1 / 2$ for $\varepsilon_{0}$ small. Arguing by contradiction, we suppose that $0<r_{*}<1 / 2$. Since (A.2) is satisfied for any $y \in \mathbb{R}^{4}$, with $r=r_{*}$ and $s=2$, Lemma A.5 implies that

$$
\|\nabla u\|_{L^{6}\left(B\left(y, r_{*} / 2\right)\right)}^{6} \leq 8\left(1+\frac{16}{r_{*}^{2}}\right)\|\nabla u\|_{L^{4}\left(B\left(y, r_{*}\right)\right)}^{4} .
$$

Since $0<r_{*}<1 / 2$, the Morrey inequality implies that

$$
\underset{B\left(y, r_{*} / 4\right)}{\mathrm{OSc}} u \leq K_{1} r_{*}^{\frac{1}{3}}\|\nabla u\|_{L^{6}\left(B\left(y, r_{*} / 2\right)\right)} \leq K_{2} r_{*}^{\frac{1}{3}}\left(1+\frac{16}{r_{*}^{2}}\right)^{\frac{1}{6}}\|\nabla u\|_{L^{4}\left(B\left(y, r_{*}\right)\right)}^{\frac{2}{3}} \leq K_{3} E(u)^{\frac{1}{3}},
$$

where we have used Lemma 2.8 for the last inequality and $K_{3}>0$ is a universal constant. Finally we notice that there exists a universal constant $\ell \in \mathbb{N}$ such that for any $x \in \mathbb{R}^{4}$, there is a collection of points $y_{1}, y_{2}, \ldots, y_{\ell} \in \mathbb{R}^{4}$ such that

$$
\underset{B\left(x, 2 r_{*}\right)}{\operatorname{Osc}} u \leq \sum_{k=1}^{\ell} \underset{B\left(y_{k}, r_{*} / 4\right)}{\operatorname{osc}} u .
$$

Thus, using (2.51), $\operatorname{osc}_{B\left(x, 2 r_{*}\right)} u \leq \ell K_{3} E(u)^{\frac{1}{3}}$. Taking $\varepsilon_{0} \leq 1 /\left(112 \ell K_{3}\right)^{3}$, we get that $\operatorname{osc}_{B\left(x, 2 r_{*}\right)} \leq 1 / 112$, which contradicts the definition of $r_{*}$. Therefore, $\operatorname{osc}_{B(x, 1 / 2)} u \leq 1 / 112$, for all $x \in \mathbb{R}^{4}$. Moreover, the same argument shows that

$$
\underset{B(y, 1 / 8)}{\mathrm{OSc}} u \leq K E(u)^{1 / 3}, \quad \text { for all } y \in \mathbb{R}^{4},
$$

and then (1.22) and (1.23) follow as before. 


\section{Properties related to the kernels and the convolution equations}

Through this section, we fix $u \in \tilde{\mathcal{E}}\left(\mathbb{R}^{N}\right) \cap U C\left(\mathbb{R}^{N}\right)$ a solution of $\left(T W_{c}\right)$ for a speed $c \in[0,1]$. We also use the notation introduced in Subsection 1.3

We start recalling the following result for $L_{c}$.

Lemma 3.1. [9, [9]. For any $c \in(0,1]$, we have

$$
\left\|L_{c} \widehat{f}\right\|_{L^{4 / 3}\left(\mathbb{R}^{2}\right)} \leq 11\|f\|_{L^{1}\left(\mathbb{R}^{2}\right)},
$$

and

$$
\left\|L_{c} \widehat{f}\right\|_{L^{2}\left(\mathbb{R}^{N}\right)} \leq K(N)\|f\|_{L^{\frac{2(2 N-1)}{2 N+3}\left(\mathbb{R}^{N}\right)}}, \quad \text { if } N \geq 3 .
$$

Proof. By the Plancherel identity, the estimate (3.2) is exactly [9, Lemma 4.3]. To prove (3.1), we note that

$$
\left\|L_{c} \widehat{f}\right\|_{L^{4 / 3}\left(\mathbb{R}^{2}\right)} \leq\left\|L_{c}\right\|_{L^{4 / 3}\left(\mathbb{R}^{2}\right)}\|\widehat{f}\|_{L^{\infty}\left(\mathbb{R}^{2}\right)} \leq\left\|L_{c}\right\|_{L^{4 / 3}\left(\mathbb{R}^{2}\right)}\|f\|_{L^{1}\left(\mathbb{R}^{2}\right)} .
$$

Then it only remains to compute $\left\|L_{c}\right\|_{L^{4 / 3}\left(\mathbb{R}^{2}\right)}$. Using polar coordinates, we have

$$
\begin{aligned}
\left\|L_{c}\right\|_{L^{4 / 3}\left(\mathbb{R}^{2}\right)}^{4 / 3} & =4 \int_{0}^{\infty} \int_{0}^{\pi / 2} \frac{r d \theta d r}{\left(r^{2}+1-c^{2} \cos ^{2}(\theta)\right)^{4} / 3}=6 \int_{0}^{\pi / 2} \frac{d \theta}{\left(1-c^{2} \cos ^{2}(\theta)\right)^{1 / 3}} \\
& \leq 6 \int_{0}^{\pi / 2} \frac{d \theta}{\left(1-\cos ^{2}(\theta)\right)^{1 / 3}}=6 \int_{0}^{\pi / 2} \frac{d \theta}{\sin ^{2 / 3}(\theta)}=3 B\left(\frac{1}{6}, \frac{1}{2}\right),
\end{aligned}
$$

where $B$ denotes the Beta function. Using that $B(x, y)=\Gamma(x) \Gamma(y) / \Gamma(x+y)$, we conclude that

$$
\left\|L_{c}\right\|_{L^{4 / 3}\left(\mathbb{R}^{2}\right)} \leq\left(3 \frac{\Gamma(1 / 6) \Gamma(1 / 2)}{\Gamma(2 / 3)}\right)^{3 / 4} \leq 11 .
$$

From (3.3) and (3.4), (3.1) follows.

Now we are able to prove the exact form of estimate (E1) stated in the introduction and also further integrability for $u_{3}$.

Proposition 3.2. Let $N \geq 2$ and $c \in(0,1)$. Then $u_{3} \in L^{p}\left(\mathbb{R}^{N}\right)$, for all $p \in(1,2)$. Moreover, if $c \in(0,1]$ and $\left\|u_{3}\right\|_{L^{\infty}\left(\mathbb{R}^{N}\right)} \leq 1 / 2$, we have

$$
\left\|u_{3}\right\|_{L^{4}\left(\mathbb{R}^{2}\right)} \leq 54\left\|u_{3}\right\|_{L^{\infty}\left(\mathbb{R}^{N}\right)} E(u),
$$

and

$$
\left\|u_{3}\right\|_{L^{2}\left(\mathbb{R}^{N}\right)} \leq K(N)\left\|u_{3}\right\|_{L^{\infty}\left(\mathbb{R}^{N}\right)}\left(1+\|\nabla u\|_{L^{\infty}\left(\mathbb{R}^{N}\right)}^{\frac{2 N-5}{2(2 N-1)}}\right) E(u)^{\frac{2 N+3}{2(2 N-1)}}, \quad \text { if } N \geq 3 .
$$

Proof. Let us recall that by Propositions 1.4 and 1.5, and noticing that

$$
G=-u_{3}^{2} \nabla \theta, \quad \text { on } B(0,3 R)^{c},
$$

we infer that $F, G_{1}, G_{2} \in L^{p}\left(\mathbb{R}^{N}\right)$, for all $p \in[1, \infty]$. On the other hand, from the Riesz-operator theory, the functions $\xi \mapsto \xi_{i} \xi_{j} /|\xi|^{2}$ are $L^{q}$-multipliers for any $q \in(1, \infty)$ and $1 \leq i, j \leq N$. Since $L_{c}$ is also an $L^{q}$-multiplier for any $q \in(1, \infty)$ (see [14), from (1.14) we conclude that $u_{3} \in L^{q}\left(\mathbb{R}^{N}\right)$, for all $q \in(1, \infty)$.

We turn now to the proof (3.5). Using (1.14) and the Hausdorff-Young inequality

$$
\|\eta\|_{L^{q}\left(\mathbb{R}^{N}\right)} \leq p^{1 / 2 p} q^{-1 / 2 q}\|\widehat{\eta}\|_{L^{p}\left(\mathbb{R}^{N}\right)}, \quad p \in[1,2], q=p /(p-1),
$$

with $p=4 / 3$, and (3.1) we obtain

$$
\left\|u_{3}\right\|_{L^{4}\left(\mathbb{R}^{2}\right)} \leq\left\|\widehat{u}_{3}\right\|_{L^{4 / 3}\left(\mathbb{R}^{2}\right)} \leq 11\left(\|F\|_{L^{1}\left(\mathbb{R}^{2}\right)}+\left\|G_{1}\right\|_{L^{1}\left(\mathbb{R}^{2}\right)}+\frac{1}{2}\left\|G_{2}\right\|_{L^{1}\left(\mathbb{R}^{2}\right)}\right),
$$

where we have used that $\xi_{1}^{2} /|\xi|^{2} \leq 1$ and $\xi_{1} \xi_{2} /|\xi|^{2} \leq\left(\xi_{1}^{2}+\xi_{2}^{2}\right) /\left(2|\xi|^{2}\right) \leq 1 / 2$ for the last inequality. 
On the other hand, since $\left\|u_{3}\right\|_{L^{\infty}\left(\mathbb{R}^{N}\right)} \leq 1 / 2$, the inequality (4.6) implies that

$$
\left\|G_{j}\right\|_{L^{1}\left(\mathbb{R}^{N}\right)} \leq \frac{2}{\sqrt{3}}\left\|u_{3}\right\|_{L^{\infty}\left(\mathbb{R}^{N}\right)} E(u) .
$$

From (3.8) and (3.9), since $F=2 e(u) u_{3}+c G_{1}$ and $11(2+5 / \sqrt{3})<54$, (3.5) follows.

Let us prove now (3.6). By applying the Plancherel identity to (1.14) and using (3.2) and (3.9), we are led to

$$
\begin{aligned}
\left\|u_{3}\right\|_{L^{2}\left(\mathbb{R}^{N}\right)} & \leq K(N)\left(\|F\|_{L^{\frac{2(2 N-1)}{2 N+3}}\left(\mathbb{R}^{N}\right)}+\sum_{j=1}^{N}\left\|G_{j}\right\|_{L^{\frac{2(2 N-1)}{2 N+3}}\left(\mathbb{R}^{N}\right)}\right) \\
& \leq K(N)\left\|u_{3} e(u)\right\|_{L^{\frac{2(2 N-1)}{2 N+3}}\left(\mathbb{R}^{N}\right)} \\
& \leq K(N)\left\|u_{3}\right\|_{L^{\infty}\left(\mathbb{R}^{N}\right)}\|e(u)\|_{L^{\infty}\left(\mathbb{R}^{N}\right)}^{\frac{2 N-5}{2(2 N-1)}}\|e(u)\|_{L^{1}\left(\mathbb{R}^{N}\right)}^{\frac{2 N+3}{2(2 N)}},
\end{aligned}
$$

which gives (3.6).

Lemma 3.3. For all $k \in \mathbb{N}$ and $p \in(1, \infty]$, we have $u_{3}, \nabla(\chi \theta) \in W^{k, p}\left(\mathbb{R}^{N}\right)$.

Proof. By Propositions 1.4 and 1.5, it remains only to treat the case $p \in(1,2)$. Differentiating (1.15) and (1.17), we have

$$
\begin{aligned}
\partial^{\alpha} u_{3} & =\mathcal{L}_{c} * \partial^{\alpha} F-c \sum_{j=1}^{N} \mathcal{L}_{c, j} * \partial^{\alpha} G_{j} \\
\partial^{\alpha} \partial_{j}(\chi \theta) & =c \mathcal{L}_{c, j} * \partial^{\alpha} F-c^{2} \sum_{k=1}^{N} \mathcal{T}_{c, j, k} * \partial^{\alpha} G_{k}-\sum_{k=1}^{N} \mathcal{R}_{j, k} * \partial^{\alpha} G_{k},
\end{aligned}
$$

for all $\alpha \in \mathbb{N}^{N}$. The conclusion follows by observing that $\mathcal{L}_{c, j}, \mathcal{T}_{c, j, k}$ and $\mathcal{R}_{j, k}$ are $L^{p}$-multipliers for all $p \in(1, \infty)$, that $u_{3}, \nabla(\chi \theta), \nabla u \in W^{k, p}\left(\mathbb{R}^{N}\right)$ for all $k \in \mathbb{N}$ and $p \in[2, \infty)$ and using the Leibniz rule.

Corollary 3.4. Let $N \geq 2$ and $c \in[0,1)$. Then the function $\theta$ is bounded on $B(0, R)^{c}$ and there exists $\bar{\theta} \in \mathbb{R}$ such that

$$
\theta(x) \rightarrow \bar{\theta}, \quad \text { as }|x| \rightarrow \infty .
$$

Proof. By Lemma 3.3, $\nabla \theta \in L^{p}\left(\mathbb{R}^{N}\right)$, for all $1<p \leq \infty$. Then there exists $\bar{\theta} \in \mathbb{R}$ such that $\theta-\bar{\theta} \in$ $L^{\frac{N p}{N-p}}\left(\mathbb{R}^{N}\right)$ (see e.g. [24, Theorem 4.5.9]). Since $\nabla \theta \in L^{\infty}\left(\mathbb{R}^{N}\right)$, we have $\theta \in U C\left(\mathbb{R}^{N}\right)$ and therefore (3.10) follows.

Proof of Proposition 1.2, For $c=0$, we deduce from (4.1) and (4.2) that $\left\|u_{3}\right\|_{L^{2}\left(\mathbb{R}^{N}\right)}=0$, so that $u_{3} \equiv 0$. Thus $\check{u}=e^{i \theta}$ on $\mathbb{R}^{N}$ and using $\left(\mathrm{TW}_{c}\right)$ (see (5.2)) we deduce that $\Delta \theta=0$ on $\mathbb{R}^{N}$. Therefore, by Corollary 3.4, we obtain that $\theta$ is a bounded harmonic function, which implies that it is constant and so that $\check{u}$ is constant.

\section{Pohozaev identities}

We start establishing the following Pohozaev identities for $\left(\mathrm{TW}_{c}\right)$. For this purpose, we introduce the notation

$$
w_{k}(v) \equiv v \cdot\left(\partial_{1} v \times \partial_{k} v\right), \quad k \in\{2, \ldots, N\} .
$$

Proposition 4.1. Let $u \in \mathcal{E}\left(\mathbb{R}^{N}\right) \cap C^{2}\left(\mathbb{R}^{N}\right)$ be a solution of $\left(\mathrm{TW}_{c}\right)$. Then there exists a sequence $r_{n} \rightarrow \infty$ such that

$$
\begin{aligned}
& E(u)=\int_{\mathbb{R}^{N}}\left|\partial_{1} u\right|^{2} d x, \\
& E(u)=\int_{\mathbb{R}^{N}}\left|\partial_{k} u\right|^{2} d x-c \lim _{r_{n} \rightarrow \infty} \int_{B\left(0, r_{n}\right)} x_{k} w_{k}(u) d x, \quad \text { for all } k \in\{2, \ldots, N\} .
\end{aligned}
$$


Proof. Taking inner product between $\left(\mathrm{TW}_{c}\right)$ and $x_{k} \partial_{k} u, 1 \leq k \leq N$, integrating by parts in the ball $B(0, R)$ and using that $u \cdot \partial_{k} u=0$, we obtain

$$
\begin{array}{r}
\int_{B(0, R)}\left|\partial_{k} u\right|^{2}-\frac{1}{2} \int_{B(0, R)}|\nabla u|^{2}-\int_{\partial B(0, R)} \frac{\partial u}{\partial \nu} \cdot \partial_{k} u x_{k}+\int_{\partial B(0, R)}|\nabla u|^{2} x_{k} \nu_{k}= \\
\frac{1}{2} \int_{B(0, R)} u_{3}^{2}-\frac{1}{2} \int_{\partial B(0, R)} u_{3}^{2} x_{k} \nu_{k}+c \int_{B(0, R)} x_{k} w_{k}(u),
\end{array}
$$

where $\nu$ denotes the exterior normal of the ball $B(0, R)$ and $\frac{\partial u}{\partial \nu}=\left(\nabla u_{1} \cdot \nu, \nabla u_{2} \cdot \nu, \nabla u_{3} \cdot \nu\right)$. By LemmaA.4. there is a sequence $r_{n} \rightarrow \infty$ such that

$$
-\int_{\partial B\left(0, r_{n}\right)} \frac{\partial u}{\partial \nu} \cdot \partial_{k} u x_{k}+\int_{\partial B\left(0, r_{n}\right)}|\nabla u|^{2} x_{k} \nu_{k}+\frac{1}{2} \int_{\partial B\left(0, r_{n}\right)} u_{3}^{2} x_{k} \nu_{k} \rightarrow 0, \quad \text { as } n \rightarrow \infty .
$$

Therefore

$$
E(u)=\int_{\mathbb{R}^{N}}\left|\partial_{k} u\right|^{2}-c \lim _{r_{n} \rightarrow \infty} \int_{B\left(0, r_{n}\right)} x_{k} w_{k}(u),
$$

which completes the proof.

Let us now discuss the definition of momentum in the two dimensional case. Formally, the first component of the vectorial momentum is given by (see [40])

$$
p(v)=-\int_{\mathbb{R}^{2}} x_{2} w_{2}(u) d x
$$

but it is not clear that this quantity is well-defined in $\mathcal{E}\left(\mathbb{R}^{2}\right)$. In general, it is a delicate task to define the momentum as a functional is the energy space. This difficulty also appears in the context of the Gross-Pitaevskii equation (see e.g. [10]). For the purpose of this paper, we will only define $p$ for smooth solutions of $\left(\mathrm{TW}_{c}\right)$. In fact, from Proposition 4.1, there exists a sequence $r_{n} \rightarrow \infty$ such that the limit

$$
\lim _{r_{n} \rightarrow \infty} \int_{B\left(0, r_{n}\right)} x_{2} x_{2} w_{2}(u) d x
$$

exists. Moreover, (4.2) shows that this limit does not depend on the sequence $r_{n}$ and therefore we will define this quantity as the momentum

$$
p(u)=-\lim _{r_{n} \rightarrow \infty} \int_{B\left(0, r_{n}\right)} x_{2} w_{2}(u) d x .
$$

With this notation we have the following consequence of Proposition 4.1

Corollary 4.2. Let $u \in \mathcal{E}\left(\mathbb{R}^{2}\right)$ be a solution of $\left(\mathrm{TW}_{c}\right.$. Then

$$
\int_{\mathbb{R}^{2}} u_{3}^{2} d x=c p(u)
$$

Proof. Writing

$$
\int_{\mathbb{R}^{2}} u_{3}^{2} d x=2 E(u)-\int_{\mathbb{R}^{2}}\left|\partial_{1} u\right|^{2} d x-\int_{\mathbb{R}^{2}}\left|\partial_{2} u\right|^{2} d x
$$

since $u \in C^{2}\left(\mathbb{R}^{2}\right)$ by Proposition 1.4, the result is a direct consequence of Proposition 4.1

In the case that $u$ admits a global lifting, we obtain

Lemma 4.3. Let $u \in \mathcal{E}\left(\mathbb{R}^{2}\right) \cap C^{2}\left(\mathbb{R}^{2}\right)$ such that $\left\|u_{3}\right\|_{L^{\infty}\left(\mathbb{R}^{2}\right)}<1$. Then

$$
p(u)=\int_{\mathbb{R}^{2}} u_{3} \partial_{1} \theta
$$

where $u_{1}+i u_{2}=\sqrt{1-u_{3}^{2}} e^{i \theta}$. 
Proof. First we notice that

$$
\left|u_{3} \partial_{1} \theta\right| \leq \frac{\left|u_{3}\right|\left|1-u_{3}^{2}\right|^{\frac{1}{2}}\left|\partial_{1} \theta\right|}{\left(1-\left\|u_{3}\right\|_{L^{\infty}\left(\mathbb{R}^{2}\right)}^{2}\right)^{1 / 2}} \leq \frac{e(u)}{\left(1-\left\|u_{3}\right\|_{L^{\infty}\left(\mathbb{R}^{2}\right)}^{2}\right)^{1 / 2}},
$$

so that the integral in (4.5) is well-defined in $\mathcal{E}\left(\mathbb{R}^{2}\right)$. We notice that

$$
u \cdot\left(\partial_{1} u \times \partial_{2} u\right)=u_{3} \varrho\left(\partial_{1} \varrho \partial_{2} \theta-\partial_{2} \varrho \partial_{1} \theta\right)+\varrho^{2}\left(\partial_{1} \theta \partial_{2} u_{3}-\partial_{2} \theta \partial_{1} u_{3}\right)=\partial_{2}\left(u_{3} \partial_{1} \theta\right)-\partial_{1}\left(u_{3} \partial_{2} \theta\right),
$$

where we have used that $u_{3}^{2}=1-\varrho^{2}$ for the last equality. Then, multiplying by $x_{2}$, integrating by parts and using the definition of $p(u)$, (4.5) follows.

From (4.6), we see that integral in (4.5) is well-defined in $\mathcal{E}\left(\mathbb{R}^{2}\right)$. Actually, integrating on $\mathbb{R}^{N}$ instead of $\mathbb{R}^{2}$, this expression provides a general definition of momentum, for functions that admit a global lifting, in any dimension. We will see this for $N=1$ in Section 6 .

\section{$5 \quad$ Properties of solutions satisfying $\left\|u_{3}\right\|_{L^{\infty}\left(\mathbb{R}^{N}\right)} \leq 1 / 2$}

In this section we assume that $u \in \tilde{\mathcal{E}}\left(\mathbb{R}^{N}\right) \cap U C\left(\mathbb{R}^{N}\right)$ is a nontrivial solution of (TW with $c \in(0,1]$ and

$$
\left\|u_{3}\right\|_{L^{\infty}\left(\mathbb{R}^{N}\right)} \leq \frac{1}{2}
$$

We have chosen $1 / 2$ to simplify the estimates. The main assumption here is that $\left\|u_{3}\right\|_{L^{\infty}\left(\mathbb{R}^{N}\right)}<1$, which implies that $\check{u}=\varrho e^{i \theta}$ on $\mathbb{R}^{N}$. Hence we can recast $\left(\mathrm{TW}_{c}\right)$ as

$$
\begin{aligned}
& \operatorname{div}\left(\varrho^{2} \nabla \theta\right)=c \partial_{1} u_{3}, \\
& -\Delta \varrho+\varrho|\nabla \theta|^{2}=2 e(u) \varrho-c u_{3} \varrho \partial_{1} \theta \\
& -\Delta u_{3}=(2 e(u)-1) u_{3}+c \varrho^{2} \partial_{1} \theta
\end{aligned}
$$

From these equations we obtain the following useful integral relations.

Lemma 5.1. We have the following identities

$$
\begin{aligned}
\int_{\mathbb{R}^{N}} \varrho^{2}|\nabla \theta|^{2} & =c \int_{\mathbb{R}^{N}} u_{3} \partial_{1} \theta, \\
\int_{\mathbb{R}^{N}}|\nabla \varrho|^{2}+\int_{\mathbb{R}^{N}} \varrho^{2}|\nabla \theta|^{2} & =2 \int_{\mathbb{R}^{N}} e(u) \varrho^{2}-c \int_{\mathbb{R}^{N}} u_{3} \varrho^{2} \partial_{1} \theta, \\
2 \int_{\mathbb{R}^{N}} \varrho|\nabla \varrho|^{2}+2 \int_{\mathbb{R}^{N}} e(u) u_{3}^{2} \varrho & =\int_{\mathbb{R}^{N}} \varrho u_{3}^{2}|\nabla \theta|^{2}+c \int_{\mathbb{R}^{N}} \varrho u_{3}^{3} \partial_{1} \theta, \\
\int_{\mathbb{R}^{N}}\left|\nabla u_{3}\right|^{2}+\int_{\mathbb{R}^{N}} u_{3}^{2} & =2 \int_{\mathbb{R}^{N}} e(u) u_{3}^{2}+c \int_{\mathbb{R}^{N}} \varrho^{2} u_{3} \partial_{1} \theta .
\end{aligned}
$$

Proof. First we recall that by Lemma A.4 for any $f \in L^{2}\left(\mathbb{R}^{2}\right)$, there exists a sequence $R_{n} \rightarrow \infty$ such that

$$
\int_{\partial B\left(0, R_{n}\right)}|f| \leq \frac{(2 \pi)^{1 / 2}}{\left(\ln \left(R_{n}\right)\right)^{1 / 2}} .
$$

Now we multiply (5.2) by $\theta$ and integrate by parts on the ball $B\left(0, R_{n}\right)$. Using the fact that $u_{3}, \nabla \theta \in$ $L^{2}\left(\mathbb{R}^{N}\right)$ and $u_{3}, \varrho, \theta \in L^{\infty}\left(\mathbb{R}^{N}\right)$, we can choose $R_{n}$ as in (5.9) such that the integrals on $\partial B\left(0, R_{n}\right)$ go to zero and (5.5) follows.

To obtain (5.6), (5.7) and (5.8), we multiply (5.3) by $\varrho$, (5.3) by $u_{3}^{2}$ and (5.4) by $u_{3}$, and proceed in a similar way.

The following result corresponds to the estimate (E2) in the case $N \geq 3$.

\section{Proposition 5.2.}

$$
E(u) \leq 3\left\|u_{3}\right\|_{L^{2}\left(\mathbb{R}^{N}\right)}^{2}
$$


Proof. Let $\delta=\left\|u_{3}\right\|_{L^{\infty}\left(\mathbb{R}^{N}\right)} \in[0,1 / 2]$. By the Cauchy-Schwarz inequality we have

$$
\int_{\mathbb{R}^{N}} u_{3} \partial_{1} \theta \leq\left(\int_{\mathbb{R}^{N}} u_{3}^{2}\right)^{\frac{1}{2}}\left(\int_{\mathbb{R}^{N}}\left(\partial_{1} \theta\right)^{2}\right)^{\frac{1}{2}} \leq \frac{1}{\sqrt{1-\delta^{2}}}\left(\int_{\mathbb{R}^{N}} u_{3}^{2}\right)^{\frac{1}{2}}\left(\int_{\mathbb{R}^{N}} \varrho^{2}|\nabla \theta|^{2}\right)^{\frac{1}{2}} .
$$

Thus from (5.5),

$$
\int_{\mathbb{R}^{N}} \rho^{2}|\nabla \theta|^{2} \leq \frac{c^{2}}{1-\delta^{2}} \int_{\mathbb{R}^{N}} u_{3}^{2} \leq \frac{4}{3} \int_{\mathbb{R}^{N}} u_{3}^{2}
$$

On the other hand, from (5.7) and the Cauchy-Schwarz inequality, we obtain

$$
2 \sqrt{1-\delta^{2}} \int_{\mathbb{R}^{N}}|\nabla \varrho|^{2} \leq \frac{\delta^{2}}{\sqrt{1-\delta^{2}}} \int_{\mathbb{R}^{N}} \varrho^{2}|\nabla \theta|^{2}+c \delta^{2}\left(\int_{\mathbb{R}^{N}} u_{3}^{2}\right)^{\frac{1}{2}}\left(\int_{\mathbb{R}^{N}} \varrho^{2}|\nabla \theta|^{2}\right)^{\frac{1}{2}} .
$$

By combining (5.11) and (5.12), with $\delta \leq 1 / 2$, we are led to

$$
\int_{\mathbb{R}^{N}}|\nabla \rho|^{2} \leq \frac{7}{18} \int_{\mathbb{R}^{N}} u_{3}^{2}
$$

From (5.8), using the Cauchy-Schwarz inequality, we have

$$
\left(1-\delta^{2}\right) \int_{\mathbb{R}^{N}}\left(\left|\nabla u_{3}\right|^{2}+u_{3}^{2}\right) \leq \delta^{2}\left(\int_{\mathbb{R}^{N}}|\nabla \rho|^{2}+\int_{\mathbb{R}^{N}} \rho^{2}|\nabla \theta|^{2}\right)+\left(\int_{\mathbb{R}^{N}} u_{3}^{2}\right)^{\frac{1}{2}}\left(\int_{\mathbb{R}^{N}} \varrho^{2}|\nabla \theta|^{2}\right)^{\frac{1}{2}},
$$

so that, by (5.11) and (5.13),

$$
\int_{\mathbb{R}^{N}}\left|\nabla u_{3}\right|^{2} \leq\left(\frac{4}{3}\left(\frac{1}{4}\left(\frac{7}{18}+\frac{4}{3}\right)+\left(\frac{4}{3}\right)^{1 / 2}\right)-1\right) \int_{\mathbb{R}^{N}} u_{3}^{2} \leq 2 \int_{\mathbb{R}^{N}} u_{3}^{2} .
$$

Finally, by putting together (5.11), (5.13) and (5.14),

$$
E(u) \leq \frac{1}{2}\left(\frac{4}{3}+\frac{7}{18}+3\right) \int_{\mathbb{R}^{N}} u_{3}^{2} \leq 3 \int_{\mathbb{R}^{N}} u_{3}^{2} .
$$

In the two-dimensional case, Corollary 4.2 allows us also to estimate the energy in terms of $\left\|u_{3}\right\|_{L^{4}\left(\mathbb{R}^{2}\right)}$. To this purpose, as remarked in [7, it is useful to study the norm of $\partial_{1} \theta-c u_{3}$.

\section{Lemma 5.3.}

$$
\left\|\partial_{1} \theta-c u_{3}\right\|_{L^{2}\left(\mathbb{R}^{2}\right)}^{2}+\left\|\partial_{2} \theta\right\|_{L^{2}\left(\mathbb{R}^{2}\right)}^{2} \leq \frac{9}{4}\left\|u_{3}\right\|_{L^{4}\left(\mathbb{R}^{2}\right)}^{4} .
$$

Proof. By adding (4.4) and (5.5), we obtain

$$
\int_{\mathbb{R}^{2}} u_{3}^{2}+\int_{\mathbb{R}^{2}} \varrho^{2}|\nabla \theta|^{2}=2 c \int_{\mathbb{R}^{2}} u_{3} \partial_{1} \theta
$$

Since $|\nabla \theta|^{2}=\left(\partial_{1} \theta\right)^{2}+\left(\partial_{2} \theta\right)^{2}$, by defining the function $\varphi=\partial_{1} \theta-c u_{3}$, we have $\partial_{1} \theta=\varphi+c u_{3}$ and then we recast (5.15) as

$$
\int_{\mathbb{R}^{2}}\left(1-u_{3}^{2}\right)\left(\varphi^{2}+\left(\partial_{2} \theta\right)^{2}\right)+\left(1-c^{2}\right) \int_{\mathbb{R}^{2}} u_{3}^{2}=2 c \int_{\mathbb{R}^{2}} u_{3}^{3} \varphi+c^{2} \int_{\mathbb{R}^{2}} u_{3}^{4}
$$

Letting $\delta=\left\|u_{3}\right\|_{L^{\infty}\left(\mathbb{R}^{2}\right)}$ and using that $c \in[0,1]$, we conclude that

$$
\left(1-\delta^{2}\right)\left(\|\varphi\|_{L^{2}\left(\mathbb{R}^{2}\right)}^{2}+\left\|\partial_{2} \theta\right\|_{L^{2}\left(\mathbb{R}^{2}\right)}^{2}\right) \leq 2\left\|u_{3}^{3} \varphi\right\|_{L^{1}\left(\mathbb{R}^{2}\right)}+\left\|u_{3}\right\|_{L^{4}\left(\mathbb{R}^{2}\right)}^{4} .
$$

For the first term in the r.h.s., we use the Hölder inequality

$$
\left\|u_{3}^{3} \varphi\right\|_{L^{1}\left(\mathbb{R}^{2}\right)} \leq\left\|u_{3}\right\|_{L^{\infty}\left(\mathbb{R}^{2}\right)}\left\|u_{3}^{2} \varphi\right\|_{L^{1}\left(\mathbb{R}^{2}\right)} \leq \delta\left\|u_{3}^{2}\right\|_{L^{2}\left(\mathbb{R}^{2}\right)}\|\varphi\|_{L^{2}\left(\mathbb{R}^{2}\right)} .
$$

Then (5.16), (5.17) and the inequality $a b \leq a^{2} / 4+b^{2}$ imply that

$$
\left(1-\delta^{2}-\frac{\delta}{2}\right)\|\varphi\|_{L^{2}\left(\mathbb{R}^{2}\right)}^{2}+\left(1-\delta^{2}\right)\left\|\partial_{2} \theta\right\|_{L^{2}\left(\mathbb{R}^{2}\right)}^{2} \leq\left(1+\frac{\delta}{4}\right)\left\|u_{3}\right\|_{L^{4}\left(\mathbb{R}^{2}\right)}^{4} .
$$

Since $\delta \leq 1 / 2$, the conclusion follows. 


\section{Lemma 5.4.}

$$
\|\nabla \varrho\|_{L^{2}\left(\mathbb{R}^{2}\right)}^{2} \leq 6\left\|u_{3}\right\|_{L^{4}\left(\mathbb{R}^{2}\right)}^{4} .
$$

Proof. Since $\varrho=\sqrt{1-u_{3}^{2}} \in[\sqrt{3} / 2,1]$, from (5.7) we have

$$
\sqrt{3} \int_{\mathbb{R}^{2}}|\nabla \varrho|^{2}+\sqrt{3} \int_{\mathbb{R}^{2}} e(u) u_{3}^{2} \leq \int_{\mathbb{R}^{2}} \varrho u_{3}^{2}\left(|\nabla \theta|^{2}+c u_{3} \partial_{1} \theta\right) .
$$

As in the proof of Lemma 5.3 , we define $\varphi=\partial_{1} \theta-c u_{3}$ so that

$$
|\nabla \theta|^{2}+c u_{3} \partial_{1} \theta=\varphi^{2}+3 c u_{3} \varphi+2 c^{2} u_{3}^{2}+\left(\partial_{2} \theta\right)^{2} .
$$

Since $\varrho \leq 1,\left|u_{3}\right| \leq 1$ and $c \leq 1$, using (5.20) and the Cauchy-Schwarz inequality,

$$
\begin{aligned}
\int_{\mathbb{R}^{2}}\left|\varrho u_{3}^{2}\left(|\nabla \theta|^{2}+c u_{3} \partial_{1} \theta\right)\right| & \leq\|\varphi\|_{L^{2}\left(\mathbb{R}^{2}\right)}^{2}+3\left\|u_{3}\right\|_{L^{4}\left(\mathbb{R}^{2}\right)}^{2}\|\varphi\|_{L^{2}\left(\mathbb{R}^{2}\right)}+2\left\|u_{3}\right\|_{L^{4}\left(\mathbb{R}^{2}\right)}^{4}+\left\|\partial_{2} \theta\right\|_{L^{2}\left(\mathbb{R}^{2}\right)}^{2} \\
& \leq \frac{35}{4}\left\|u_{3}\right\|_{L^{4}\left(\mathbb{R}^{2}\right)}^{4},
\end{aligned}
$$

where we have used Lemma 5.3 for the last inequality. By combining (5.19), (5.21) and the fact that $35 /(4 \sqrt{3}) \leq 6$, we obtain (5.18) .

Finally, we get estimate (E2) for $N=2$.

\section{Proposition 5.5.}

$$
E(u) \leq 10\left\|u_{3}\right\|_{L^{4}\left(\mathbb{R}^{2}\right)}^{4}
$$

Proof. From (5.6) we obtain

$$
2 \int_{\mathbb{R}^{2}} e(u) \varrho^{2}=\int_{\mathbb{R}^{2}}|\nabla \varrho|^{2}+\int_{\mathbb{R}^{2}} \varrho^{2}\left(|\nabla \theta|^{2}+c u_{3} \partial_{1} \theta\right)
$$

By using Lemma 5.4 (5.21) and the fact that $\varrho^{2} \in[3 / 4,1]$, we conclude that

$$
E(u) \leq \frac{59}{6}\left\|u_{3}\right\|_{L^{4}\left(\mathbb{R}^{2}\right)}^{4} \leq 10\left\|u_{3}\right\|_{L^{4}\left(\mathbb{R}^{2}\right)}^{4}
$$

At this point we dispose of all the elements to prove our result, as was sketched in the introduction.

Proof of Theorem 1.1. By virtue of Propositions 1.4 and 1.5, we can fix $\varepsilon_{0}>0$ such that if $E(u) \leq \varepsilon_{0}$, then $\left\|u_{3}\right\|_{L^{\infty}\left(\mathbb{R}^{N}\right)} \leq 1 / 2$ and $\|\nabla u\|_{L^{\infty}\left(\mathbb{R}^{N}\right)}$ is uniformly bounded. Then Propositions 3.2, 5.2] and [5.5]imply that

$$
E(u) \leq 10\left\|u_{3}\right\|_{L^{4}\left(\mathbb{R}^{N}\right)}^{4} \leq 10(54)^{4} E(u)^{4}, \quad \text { if } N=2,
$$

and

$$
E(u) \leq 3\left\|u_{3}\right\|_{L^{2}\left(\mathbb{R}^{N}\right)}^{2} \leq K E(u)^{\frac{2 N+3}{2 N-1}}, \quad \text { if } N \in\{3,4\} .
$$

Thus, since $u$ is nonconstant, $E(u)>0$ and we can divide by $E(u)$. Therefore, from (5.22) and (5.23) we conclude that $\tilde{K} \leq E(u)$, for some constant $\tilde{K}>0$. Taking $\mu=\min \left\{\varepsilon_{0}, \tilde{K}\right\}$, the proof is complete.

\section{The one-dimensional case}

In this section we consider the case $N=1$. Then equation $\left(\mathrm{TW}_{c}\right)$ is integrable and the solutions can be computed explicitly as was noticed in [36, 39, 44, More precisely, we have

Proposition 6.1. Let $N=1, c \geq 0$ and $u \in \mathcal{E}(\mathbb{R})$ be solution of $\left(\mathrm{TW}_{c}\right.$.

(i) If $c \geq 1$, then $u$ is a trivial solution. 
(ii) If $0 \leq c<1$ and $u$ is nontrivial, then, up to invariances, $u$ is given by

$$
\begin{aligned}
& u_{1}=c \operatorname{sech}\left(\sqrt{1-c^{2}} x\right), \\
& u_{2}=\tanh \left(\sqrt{1-c^{2}} x\right), \\
& u_{3}=\sqrt{1-c^{2}} \operatorname{sech}\left(\sqrt{1-c^{2}} x\right) .
\end{aligned}
$$

(iii) If $0<c<1$, we can write

$$
\check{u}=\sqrt{1-u_{3}^{2}} \exp (i \theta)
$$

where

$$
\theta=\arctan \left(\frac{\sinh \left(\sqrt{1-c^{2}} x\right)}{c}\right)
$$

Proof. We first remark that since $N=1$, it is simply to verify that $u$ is smooth and then the condition $u \in \mathcal{E}(\mathbb{R})$ implies that $u^{\prime}$ and $u_{3}$ vanish at infinity. Let us write $\left(\mathrm{TW}_{c}\right)$ in coordinates

$$
\begin{aligned}
& -u_{1}^{\prime \prime}=2 e(u) u_{1}+c\left(u_{2} u_{3}^{\prime}-u_{3} u_{2}^{\prime}\right), \\
& -u_{2}^{\prime \prime}=2 e(u) u_{2}+c\left(u_{3} u_{1}^{\prime}-u_{1} u_{3}^{\prime}\right), \\
& -u_{3}^{\prime \prime}=2 e(u) u_{3}-u_{3}+c\left(u_{1} u_{2}^{\prime}-u_{2} u_{1}^{\prime}\right) .
\end{aligned}
$$

Also, as in (1.11), we have

$$
\left(u_{1} u_{2}^{\prime}-u_{1}^{\prime} u_{2}\right)^{\prime}=c u_{3}^{\prime} .
$$

Integrating (6.9), we obtain

$$
u_{1} u_{2}^{\prime}-u_{1}^{\prime} u_{2}=c u_{3}
$$

Then, replacing (6.9) in (6.8), we get

$$
u_{3}^{\prime \prime}+2 e(u) u_{3}-\left(1-c^{2}\right) u_{3}=0 .
$$

Now, multiplying (6.6), (6.7), (6.11) by $u_{1}^{\prime}, u_{2}^{\prime}, u_{3}^{\prime}$, respectively, adding these relations and using again (6.10),

$$
-\left(\left|u^{\prime}\right|^{2}\right)^{\prime}=2 e(u)\left(u_{1}^{2}+u_{2}^{2}+u_{3}^{2}\right)^{\prime}-\left(u_{3}^{2}\right)^{\prime} .
$$

Since $\left(u_{1}^{2}+u_{2}^{2}+u_{3}^{2}\right)^{\prime}=\left(|u|^{2}\right)^{\prime}=0$, integrating (6.12) we conclude that

$$
\left|u^{\prime}\right|^{2}=u_{3}^{2}
$$

so that $e(u)=u_{3}^{2}$ and equation (6.11) reduces to

$$
u_{3}^{\prime \prime}-2 u_{3}^{3}-\left(1-c^{2}\right) u_{3}=0 .
$$

As before, multiplying (6.14) by $u_{3}^{\prime}$ and integrating, we conclude that

$$
\left(u_{3}^{\prime}\right)^{2}=u_{3}^{2}\left(\left(1-c^{2}\right)-u_{3}^{2}\right)
$$

If $u_{3}$ is identically zero, (6.13) implies that $u$ is a trivial solution. Therefore, we suppose from now on that $u_{3}$ not identically zero. Since equation (6.14) is invariant under translation, we can assume that

$$
\left|u_{3}(0)\right|=\max \left\{\left|u_{3}(x)\right|: x \in \mathbb{R}\right\}>0 \text {. }
$$

Therefore

$$
u_{3}^{\prime}(0)=0,
$$

and from (6.15) and (6.16),$u_{3}^{2}(0)=1-c^{2}$. In particular we deduce that if $c \geq 1, u_{3} \equiv 0$, which implies that $u_{1}$ and $u_{2}$ are constant, which completes the proof of (i). If $0 \leq c<1$, by the Cauchy-Lipschitz theorem, equation (6.14) with initial conditions (6.16) and $u_{3}(0)=\sqrt{1-c^{2}}$ or $u_{3}(0)=-\sqrt{1-c^{2}}$ has a unique maximal solution. It is straightforward to check that

$$
u_{3}(x)= \pm \sqrt{1-c^{2}} \operatorname{sech}\left(\sqrt{1-c^{2}} x\right)
$$


is the desired solution. Moreover, (6.17) shows that $\left\|u_{3}\right\|_{L^{\infty}(\mathbb{R})}<1$ if $c \in(0,1)$. Hence, for $c \in(0,1)$, we can write $\check{u}=\left(1-u_{3}^{2}\right)^{1 / 2} e^{i \theta}$, and then (6.9) yields

$$
\theta^{\prime}=\frac{c u_{3}}{1-u_{3}^{2}}
$$

From (6.17) and (6.18), we are led to

$$
\theta=\theta_{0}+\arctan \left(\frac{\sinh \left(\sqrt{1-c^{2}} x\right)}{c}\right)
$$

for some constant $\theta_{0} \in \mathbb{R}$, which proves (6.4)-(6.5). Using some standard identities for trigonometric and hyperbolic functions, we also obtain (6.1)-(6.3), for $c \in(0,1)$. It only remains to show that for $c=0$, (6.1) and (6.2) are the unique solutions of (6.6) -(6.8). Indeed, since $e(u)(x)=u_{3}^{2}(x)=\operatorname{sech}^{2}(x)$, we recast (6.1) and (6.2) as

$$
-\check{u}^{\prime \prime}=2 \operatorname{sech}^{2}(x) \check{u},
$$

and from (6.13) we can assume that, up to a multiplication by a complex number of modulus one,

$$
\check{u}^{\prime}(0)=1 .
$$

Then the Cauchy-Lipschitz theorem provides the existence of a unique solution of (6.19)-(6.20) in a neighborhood of $x=0$, and it is immediate to check that $\check{u}(x)=\tanh (x)$ is the solution, which concludes the proof.

In the one-dimensional case, the momentum is formally given by

$$
p(u)=\int_{\mathbb{R}} \frac{u_{3}\left(u_{1} u_{2}^{\prime}-u_{2} u_{1}^{\prime}\right)}{1-u_{3}^{2}} .
$$

If $\left\|u_{3}\right\|_{L^{\infty}(\mathbb{R})}<1$, we see that

$$
p(u)=\int_{\mathbb{R}} u_{3} \theta^{\prime},
$$

and therefore it agrees with the corresponding expression in dimension two.

Corollary 6.2. Assume that $c \in[0,1)$ and let $u \in \mathcal{E}(\mathbb{R})$ be a nontrivial solution of $\left(\mathrm{TW}_{c}\right.$ ). Then

$$
E(u)=2 \sqrt{1-c^{2}} .
$$

Moreover,

$$
p(u)=\int_{\mathbb{R}} u_{3} \theta^{\prime}=2 \arctan \left(\frac{\sqrt{1-c^{2}}}{c}\right), \quad \text { for } c \in(0,1) .
$$

In particular, we can write explicitly $E$ as a function of $p$ as

$$
E(p)=2 \sin \left(\frac{p}{2}\right)
$$

and

$$
\frac{d E}{d p}=\cos \left(\frac{p}{2}\right)=c
$$

for $c \in(0,1)$.

Proof. Using (6.3) and (6.13), we have

$$
E(u)=\int_{\mathbb{R}} u_{3}^{2}=\sqrt{1-c^{2}} \int_{\mathbb{R}} \operatorname{sech}^{2}(x) d x=2 \sqrt{1-c^{2}} .
$$

For the momentum, (6.18) yields

$$
p(u)=\int_{\mathbb{R}} u_{3} \theta^{\prime}=c \int_{\mathbb{R}} \frac{u_{3}^{2}}{1-u_{3}^{2}}=c\left(1-c^{2}\right) \int_{\mathbb{R}} \frac{\operatorname{sech}^{2}\left(\sqrt{1-c^{2}} x\right)}{1-\left(1-c^{2}\right) \operatorname{sech}^{2}\left(\sqrt{1-c^{2}} x\right)} d x .
$$


Then, using the change of variables $y=\frac{\sqrt{1-c^{2}}}{c} \tanh \left(\sqrt{1-c^{2}} x\right)$, we obtain (6.22), from where we deduce that

$$
c^{2}=\frac{1}{\tan ^{2}(p / 2)+1}=\cos ^{2}(p / 2) \text {. }
$$

Finally, from (6.21) and (6.25), we establish (6.23), from where (6.24) is an immediate consequence.

Proposition 1.3 follows from Proposition 6.1 and Corollary 6.2.

\section{Decay at infinity}

In this section we provide a sketch the proof of Theorem 1.6. The first step is to obtain some algebraic decay at infinity of the solutions of $\left(\mathrm{TW}_{c}\right)$. This can be achieved following an argument of [3].

Proposition 7.1. Assume that $c \in(0,1)$. Let $u \in \mathcal{E}\left(\mathbb{R}^{N}\right)$ be a solution of $\left(\mathrm{TW}_{c}\right)$. Suppose further that $u \in U C\left(\mathbb{R}^{N}\right)$ if $N \geq 3$. Then there exist constants $R_{1}, \alpha>0$ such that for all $R \geq R_{1}$,

$$
\int_{B(0, R)^{c}} e(u) \leq\left(\frac{R_{1}}{R}\right)^{\alpha} \int_{B\left(0, R_{1}\right)^{c}} e(u) .
$$

Proof. By Corollary 2.5, there exists $R_{0}>0$ such that equations (5.2)-(5.4) hold on $B\left(0, R_{0}\right)^{c}$. Let $\rho>r \geq R_{0}$ and

$$
\Omega_{r, \rho}=\{r \leq|x| \leq \rho\} .
$$

Multiplying (5.2) by $\theta-\theta_{r}$, with $\theta_{r}=\frac{1}{\left|\partial B_{r}\right|} \int_{\partial B_{r}} \theta$, and integrating by parts, we get

$$
\int_{\Omega_{r, \rho}} \varrho^{2} \nabla \theta^{2}=c \int_{\Omega_{r, \rho}} u_{3} \partial_{1} \theta+\int_{\partial \Omega_{r, \rho}}\left(\theta-\theta_{r}\right) \varrho^{2} \partial_{\nu} \theta-c \int_{\partial \Omega_{r, \rho}}\left(\theta-\theta_{r}\right) u_{3} \nu_{1},
$$

where $\nu$ denotes the outward normal to $\Omega_{r, \rho}$.

We recall that the Poincaré inequality for $\partial B_{r}$ reads

$$
\int_{\partial B_{r}}\left(\theta-\theta_{r}\right)^{2} \leq r^{2} \int_{\partial B_{r}}\left|\nabla_{\tau} \theta\right|^{2}
$$

Then we obtain

$$
\left|\int_{\partial B_{r}}\left(\theta-\theta_{r}\right) \varrho^{2} \partial_{\nu} \theta\right| \leq r\left(\int_{\partial B_{r}}|\nabla \theta|^{2}\right)^{1 / 2}\left(\int_{\partial B_{r}}|\rho \nabla \theta|^{2}\right)^{1 / 2} \leq \frac{r}{\sqrt{1-\delta^{2}}} \int_{\partial B_{r}}|\rho \nabla \theta|^{2},
$$

where $\delta=\left\|u_{3}\right\|_{L^{\infty}\left(B_{r}^{c}\right)}$. Similarly, using also the inequality $a b \leq a^{2} / 2+b^{2} / 2$,

$$
\left|\int_{\partial \Omega_{r, \rho}}\left(\theta-\theta_{r}\right) u_{3} \nu_{1}\right| \leq \frac{r}{\sqrt{1-\delta^{2}}} \int_{\partial B_{r}} e(u) \quad \text { and } \quad\left|\int_{B_{r}^{c}} u_{3} \partial_{1} \theta\right| \leq \frac{1}{\sqrt{1-\delta^{2}}} \int_{B_{r}^{c}} e(u) .
$$

On the other hand, by Lemma 3.3 and Corollary 3.4 .

$$
\left(\theta-\theta_{r}\right) \varrho^{2} \partial_{\nu} \theta,\left(\theta-\theta_{r}\right) u_{3} \nu_{1} \in L^{2}\left(B\left(0, R_{0}\right)^{c}\right)
$$

Then by Lemma A.4 we conclude that there exists a sequence $\rho_{n} \rightarrow \infty$ such that

$$
\int_{\partial B_{\rho_{n}}}\left(\theta-\theta_{r}\right) \varrho^{2} \partial_{\nu} \theta \rightarrow 0 \text { and } \int_{\partial B_{\rho_{n}}}\left(\theta-\theta_{r}\right) u_{3} \nu_{1} \rightarrow 0 .
$$

Therefore, taking $\rho=\rho_{n}$, using (7.2) -(7.3) and the dominated convergence theorem we conclude that

$$
\int_{B_{r}^{c}} \varrho^{2} \nabla \theta^{2} \leq \frac{c}{\sqrt{1-\delta^{2}}} \int_{B_{r}^{c}} e(u)+\frac{3 r}{\sqrt{1-\delta^{2}}} \int_{\partial B_{r}} e(u) .
$$


In the same way, multiplying (5.4) by $u_{3}$, integrating by parts on the set $\Omega_{r, \tilde{\rho}_{n}}$, for a suitable sequence $\tilde{\rho}_{n} \rightarrow \infty$, we are led to

$$
\int_{B_{r}^{c}}\left(\left|\nabla u_{3}\right|^{2}+u_{3}^{2}\right) \leq\left(2 \delta^{2}+c\right) \int_{B_{r}^{c}} e(u)+\int_{\partial B_{r}} e(u) .
$$

Since $c<1$, we can choose $r$ large enough such that

$$
\frac{1}{2\left(1-\delta^{2}\right)}\left(2 \delta^{2}+c\left(1+\frac{1}{\sqrt{1-\delta^{2}}}\right)\right)<1 .
$$

Therefore, noticing that

$$
e(u) \leq \frac{1}{2\left(1-\delta^{2}\right)}\left(\left|\nabla u_{3}\right|^{2}+\varrho^{2}|\nabla \theta|^{2}+u_{3}^{2}\right),
$$

we conclude that there exists a constant $K(\delta, c)>0$ such that

$$
\int_{B_{r}^{c}} e(u) \leq K(\delta, c) r \int_{\partial B_{r}} e(u) .
$$

Since

$$
\frac{d}{d r} \int_{B_{r}^{c}} e(u)=-\int_{\partial B_{r}} e(u),
$$

we can integrate inequality (7.4) to conclude that

$$
\int_{B_{R}^{c}} e(u) \leq\left(\frac{r}{R}\right)^{1 / K(c, \delta)} \int_{B_{r}^{c}} e(u), \quad \text { for all } R \geq r,
$$

which completes the proof.

Corollary 7.2. Under the hypotheses and notations of Proposition 7.1, we have

$$
|\cdot|^{\beta} e(u) \in L^{1}\left(\mathbb{R}^{N}\right) \quad \text { and } \quad|\cdot|^{\beta}\left(|F|+\left|G_{1}\right|+\cdots+\left|G_{N}\right|\right) \in L^{1}\left(\mathbb{R}^{N}\right),
$$

for all $\beta \in[0, \alpha)$.

Proof. Since $u \in C^{\infty}\left(\mathbb{R}^{N}\right)$, the fact that $|\cdot|^{\beta} e(u) \in L^{1}\left(\mathbb{R}^{N}\right)$ is a direct consequence of Proposition 7.1 (see e.g. [14, Proposition 28]). On the other hand, we take $R$ large enough such that $\left\|u_{3}\right\|_{L^{\infty}\left(B_{R}^{c}\right)} \leq 1 / 2$. Then using that $\left|u_{3}\right| \leq 1$, (3.7) and (4.6), we deduce that for all $j \in\{1, \ldots, N\}$,

$$
|F|+\left|G_{j}\right| \leq 2 e(u)+\left|u_{3}^{2} \partial_{1} \theta\right|+\left|u_{3}^{2} \partial_{j} \theta\right| \leq \frac{|\nabla \theta|^{2}}{2} \leq\left(2+\frac{4}{\sqrt{3}}\right) e(u),
$$

and then the conclusion follows.

The properties of the kernels appearing in equations (1.15) and (1.17) has been extensively studied in [14]. Indeed, using the sets

$$
\begin{aligned}
\mathcal{M}_{k}\left(\mathbb{R}^{N}\right) & =\left\{f: \mathbb{R}^{N} \rightarrow \mathbb{C}: \sup _{x \in \mathbb{R}^{N}}|x|^{k}|f(x)|<\infty\right\}, \quad k \in \mathbb{N}, \\
\mathcal{M}\left(\mathbb{R}^{N}\right) & =\left\{f \in C^{\infty}\left(\mathbb{R}^{N} \backslash\{0\} ; \mathbb{C}\right): D^{k} f \in \mathcal{M}_{k}\left(\mathbb{R}^{N}\right) \cap \mathcal{M}_{k+2}\left(\mathbb{R}^{N}\right), \text { for all } k \in \mathbb{N}\right\},
\end{aligned}
$$

it is proved that

$$
D^{n} \mathcal{L}_{c}, D^{n} \mathcal{L}_{c, j}, D^{n} \mathcal{T}_{c, j, k} \in \mathcal{M}_{\alpha+n}\left(\mathbb{R}^{N}\right), \text { for all } 1 \leq j, k \leq N, n \in \mathbb{N}, \alpha \in(N-2, N],
$$

and also that

$$
\widehat{\mathcal{L}}_{c}, \widehat{\mathcal{L}}_{c, j}, \widehat{\mathcal{T}}_{c, j, k} \in \mathcal{M}\left(\mathbb{R}^{N}\right) .
$$

Similar results hold for the composed Riesz kernels $\mathcal{R}_{j, k}$. By combining these results with Corollary 7.2 , equations (1.15) and (1.17) allow us to obtain the following algebraic decay. 
Lemma 7.3. For any $n \in \mathbb{N}$,

$$
u_{3}, D^{n}(\nabla(\chi \theta)), D^{n}(\nabla \check{u}) \in \mathcal{M}_{N}\left(\mathbb{R}^{N}\right) \quad \text { and } \quad D^{n} u_{3} \in \mathcal{M}_{N+1}\left(\mathbb{R}^{N}\right) .
$$

Proof. In view of Corollary [7.2, the proof follows using the same arguments in [14, Theorem 11].

Proposition 7.4. Let $N \geq 2$ and $c \in(0,1)$. Assume that $u \in \mathcal{E}\left(\mathbb{R}^{N}\right)$ is a solution of $\left(\mathrm{TW}_{c}\right.$ ). Suppose further that $u \in U C\left(\mathbb{R}^{N}\right)$ if $N \geq 3$. Then there exist constants $R(u), K(c, u) \geq 0$ such that

$$
\begin{aligned}
\left|u_{3}(x)\right|+|\nabla \theta(x)|+|\nabla \check{u}(x)| & \leq \frac{K(c, u)}{1+|x|^{N}}, \\
\left|\nabla u_{3}(x)\right|+\left|D^{2} \theta(x)\right|+\left|D^{2} \check{u}(x)\right| & \leq \frac{K(c, u)}{1+|x|^{N+1}}, \\
\left|D^{2} u_{3}(x)\right| & \leq \frac{K(c, u)}{1+|x|^{N+2}},
\end{aligned}
$$

for all $x \in B(0, R(u))^{c}$.

Proof. Inequality (7.7) and the estimate for $\nabla u_{3}$ in (7.8) are particular cases of Lemma 7.3 A sightly improvement of Lemma 7.3 is necessary for the decay of the second derivatives in (7.8) and (7.9). This can be done by following the lines in [15, Theorem 6], which completes the proof.

The pointwise convergence at infinity follows from general arguments in [15, valid for all functions satisfying (7.6).

Lemma 7.5 (15]). Assume that $T$ is a tempered distribution whose Fourier transform $\widehat{T}=P / Q$ is a rational fraction which belongs to $\mathcal{M}\left(\mathbb{R}^{N}\right)$ and such that $Q \neq 0$ on $\mathbb{R}^{N} \backslash\{0\}$. Then there exists a function $T_{\infty} \in L^{\infty}\left(\mathbb{S}^{N-1} ; \mathbb{C}\right)$ such that

$$
R^{N} T(R \sigma) \rightarrow T_{\infty}(\sigma), \quad \text { as } R \rightarrow \infty, \text { for all } \sigma \in \mathbb{S}^{N-1} .
$$

Moreover, assume that $f \in C^{\infty}\left(\mathbb{R}^{N}\right) \cap L^{\infty}\left(\mathbb{R}^{N}\right) \cap \mathcal{M}_{2 N}\left(\mathbb{R}^{N}\right)$. Then $g \equiv T * f$ satisfies

$$
R^{N} g(R \sigma) \rightarrow T_{\infty}(\sigma) \int_{\mathbb{R}^{N}} f(x) d x, \quad \text { as } R \rightarrow \infty, \text { for all } \sigma \in \mathbb{S}^{N-1} .
$$

Roughly speaking, it only remains to pass to the limit in the terms associated to the Riesz kernels $\mathcal{R}_{i, j}$. For this purpose, we also recall the following.

Lemma 7.6 ([15]). Assume that $f \in C^{\infty}\left(\mathbb{R}^{N}\right) \cap L^{\infty}\left(\mathbb{R}^{N}\right) \cap \mathcal{M}_{2 N}\left(\mathbb{R}^{N}\right)$ with $\nabla f \in L^{\infty}\left(\mathbb{R}^{N}\right) \cap \mathcal{M}_{2 N+1}\left(\mathbb{R}^{N}\right)$. Then $g \equiv \mathcal{R}_{j, k} * f$ satisfies for all $j, k \in\{1, \ldots, N\}$,

$$
R^{N} g(R \sigma) \rightarrow(2 \pi)^{-\frac{N}{2}} \Gamma\left(\frac{N}{2}\right)\left(\delta_{j, k}-N \sigma_{j} \sigma_{k}\right) \int_{\mathbb{R}^{N}} f(x) d x, \quad \text { as } R \rightarrow \infty, \text { for all } \sigma \in \mathbb{S}^{N-1} .
$$

Finally, we have all the elements to provide the sketch of the proof of Theorem 1.6.

Proof of Theorem 1.6. In view of (1.15), (7.6) and Lemma 7.3, we can apply Lemma 7.5 to the function $u_{3}$ to conclude that there exists $u_{3, \infty} \in L^{\infty}\left(\mathbb{S}^{N-1} ; \mathbb{R}\right)$ such that

$$
R^{N} u_{3}(R \sigma) \rightarrow u_{3, \infty}(\sigma), \quad \text { as } R \rightarrow \infty, \text { for all } \sigma \in \mathbb{S}^{N-1},
$$

where

$$
u_{3, \infty}(\sigma)=\mathcal{L}_{c, \infty}(\sigma) \int_{\mathbb{R}^{N}} F-c \sum_{j=1}^{N} \mathcal{L}_{c, j, \infty}(\sigma) \int_{\mathbb{R}^{N}} G_{j},
$$

for some functions $\mathcal{L}_{c, \infty}, \mathcal{L}_{c, j, \infty}$. Moreover, adapting [16, Proposition 2], we obtain

$$
\begin{aligned}
\mathcal{L}_{c, \infty}(\sigma) & =\frac{\Gamma\left(\frac{N}{2}\right)\left(1-c^{2}\right)^{\frac{N-3}{2}} c^{2}}{2 \pi^{\frac{N}{2}}\left(1-c^{2}+c^{2} \sigma_{1}^{2}\right)^{\frac{N}{2}}}\left(1-\frac{N \sigma_{1}^{2}}{1-c^{2}+c^{2} \sigma_{1}^{2}}\right) \\
\mathcal{L}_{c, j, \infty}(\sigma) & =\frac{\Gamma\left(\frac{N}{2}\right)\left(1-c^{2}\right)^{\frac{N-1}{2}}}{2 \pi^{\frac{N}{2}}\left(1-c^{2}+c^{2} \sigma_{1}^{2}\right)^{\frac{N}{2}}}\left(\delta_{j, 1}\left(1-c^{2}\right)^{-\frac{\delta_{j, 1}+1}{2}}-\frac{N\left(1-c^{2}\right)^{-\delta_{j, 1}} \sigma_{1} \sigma_{j}}{1-c^{2}+c^{2} \sigma_{1}^{2}}\right),
\end{aligned}
$$


which gives (1.27).

Now we turn to equation (1.17). Proceeding as before and using also Lemma 7.6, we infer that there exist functions $\theta_{\infty}^{j} \in L^{\infty}\left(\mathbb{S}^{N-1} ; \mathbb{R}\right), j \in\{1, \ldots, N\}$ such that

$$
R^{N} \partial_{j} \theta(R \sigma) \rightarrow \theta_{\infty}^{j}(\sigma), \quad \text { as } R \rightarrow \infty,
$$

for all $j \in\{1, \ldots, N\}$, and also that $\theta_{\infty}^{j}$ is given by

$$
\theta_{\infty}^{j}(\sigma)=c \mathcal{L}_{c, j, \infty}(\sigma) \int_{\mathbb{R}^{N}} F-\sum_{k=1}^{N}\left(c^{2} \mathcal{T}_{c, j, k, \infty}(\sigma)+\frac{\Gamma\left(\frac{N}{2}\right)}{2 \pi^{\frac{N}{2}}}\left(\delta_{j, k}-N \sigma_{j} \sigma_{k}\right)\right) \int_{\mathbb{R}^{N}} G_{k} .
$$

As before, adapting [16, Proposition 2] we have

$$
\begin{array}{r}
\mathcal{T}_{c, j, k, \infty}=\frac{\Gamma\left(\frac{N}{2}\right)}{2 \pi^{\frac{N}{2}} c^{2}}\left(\left(1-c^{2}\right)^{\frac{N}{2}}\left(\frac{\delta_{j, k}\left(1-c^{2}\right)^{-\frac{\delta_{j, 1}+\delta_{k, 1}+1}{2}}}{\left(1-c^{2}+c^{2} \sigma_{1}^{2}\right)^{\frac{N}{2}}}-\frac{N\left(1-c^{2}\right)^{-\delta_{j, 1}-\delta_{k, 1}+\frac{1}{2}} \sigma_{j} \sigma_{k}}{\left(1-c^{2}+c^{2} \sigma_{1}^{2}\right)^{\frac{N+2}{2}}}\right)\right. \\
\left.-\delta_{j, k}+N \sigma_{j} \sigma_{k}\right) .
\end{array}
$$

At this stage, we invoke Corollary 3.4 and suppose that $\bar{\theta}=0$. Then by [15, Lemma 10],

$$
R \theta(R \sigma) \rightarrow \theta_{\infty}(\sigma) \equiv-\frac{1}{N-1} \sum_{j=1}^{N} \sigma_{j} \theta_{\infty}^{j}, \quad \text { as } R \rightarrow \infty .
$$

A further analysis shows that the convergence in (7.10) and (7.16) are uniform, which implies that

$$
R^{N-1}(\check{u}(R \sigma)-1)=R^{N-1}\left(\sqrt{1-u_{3}^{2}(R \sigma)} \exp (i \theta(R \sigma))-1\right) \rightarrow i \theta_{\infty}(\sigma), \quad \text { in } L^{\infty}\left(\mathbb{S}^{N-1}\right) .
$$

By combining with the expression for $\theta_{\infty}^{j}$ above, (1.24) follows with $\lambda_{\infty}=1$ and $\check{u}_{\infty}=\theta_{\infty}$, provided that $\bar{\theta}=0$. Moreover, using (7.11) $-(7.16)$ and that

$$
\begin{aligned}
& \sum_{j=1}^{N} \sigma_{j} \mathcal{L}_{c, j, \infty}(\sigma)=-\frac{\Gamma\left(\frac{N}{2}\right)(N-1)\left(1-c^{2}\right)^{\frac{N-3}{2}} \sigma_{1}}{2 \pi^{\frac{N}{2}}\left(1-c^{2}+c^{2} \sigma_{1}^{2}\right)^{\frac{N}{2}}} \\
& \sum_{j=1}^{N} \sigma_{j} \mathcal{T}_{c, j, k, \infty}(\sigma)=-\frac{\Gamma\left(\frac{N}{2}\right)(N-1) \sigma_{k}}{2 \pi^{\frac{N}{2}} c^{2}}\left(\frac{\left(1-c^{2}\right)^{\frac{N}{2}-\frac{1}{2}-\delta_{k, 1}}}{\left(1-c^{2}+c^{2} \sigma_{1}^{2}\right)^{\frac{N}{2}}}-1\right),
\end{aligned}
$$

we obtain (1.26).

In the case that $\bar{\theta} \neq 0$, it is enough to redefine the function $G$ in (1.7) as

$$
G=u_{1} \nabla u_{2}-u_{2} \nabla u_{1}-\nabla(\chi(\theta-\bar{\theta})),
$$

since then we can establish an equation such as (1.17) for $\partial_{j}(\chi(\theta-\bar{\theta}))$. Since $\theta(x)-\bar{\theta} \rightarrow 0$, as $x \rightarrow \infty$, we conclude as before that there exists $\theta_{\infty} \in L^{\infty}\left(\mathbb{S}^{N-1} ; \mathbb{R}\right)$ such that

$$
R^{N-1}\left(\sqrt{1-u_{3}^{2}(R \sigma)} \exp (i(\theta(R \sigma)-\bar{\theta})-1) \rightarrow i \theta_{\infty}(\sigma), \quad \text { in } L^{\infty}\left(\mathbb{S}^{N-1}\right) .\right.
$$

Since $\sqrt{1-u_{3}^{2}(R \sigma)} \exp \left(i(\theta(R \sigma)-\bar{\theta})=\check{u}(R \sigma) \exp (-i \bar{\theta})\right.$, taking $\lambda_{\infty}=\exp (i \bar{\theta})$, we conclude that

$$
R^{N-1}\left(\check{u}(R \sigma)-\lambda_{\infty}\right) \rightarrow i \lambda_{\infty} \theta_{\infty}, \quad \text { in } L^{\infty}\left(\mathbb{S}^{N-1}\right),
$$

which completes the proof of Theorem 1.6

Acknowledgments. The author is grateful to N. Papanicolaou and S. Komineas for interesting and helpful discussions. 


\section{Appendix}

For the convenience of the reader we recall some well-known results used in this paper. We assume $\Omega$ to be a smooth open bounded domain of $\mathbb{R}^{N}$.

Theorem A.1 ([45, 30]). Let $u \in H^{1}(\Omega)$, such that $\Delta u=0$ on $D^{\prime}(\Omega)$. Then there are constants $0<\alpha \leq 1, \alpha=\alpha(N)$, and $K>0$ such that if $x \in \Omega$ and $0<\rho<r<\operatorname{dist}(x, \Omega)$,

$$
\underset{B_{\rho}}{\operatorname{Osc}} u \leq K\left(\frac{\rho}{r}\right)^{\alpha} \frac{\|u\|_{L^{2}\left(B_{r}\right)}}{r^{N / 2}} .
$$

Moreover, if $N=2$, then

$$
\underset{B_{\rho}}{\operatorname{osc}} u \leq K(\ln (\rho / r))^{-1 / 2}\|\nabla u\|_{L^{2}\left(B_{r}\right)},
$$

for some $K>0$.

Theorem A.2 (45]). Let $p>N / 2$ and $f \in L^{p}(\Omega)$. Assume that $u \in H_{0}^{1}(\Omega)$ is solution of $-\Delta u=f$, in

$\Omega$. Then $u$ is Hölder continuous in $\bar{\Omega}$. Moreover, for $\rho>0$, there exists a constant $K(\rho)$ such that

$$
\underset{B_{\rho} \cap \Omega}{\operatorname{Osc}} u \leq K(\rho)\|f\|_{L^{p}(\Omega)} .
$$

Lemma A.3. Let $f \in L^{1}\left(\mathbb{R}^{N}\right)$. Then for every $\varepsilon>0$ there exists a constant $K(\varepsilon)$ such that $f=f_{1}+f_{2}$ a.e. on $\mathbb{R}^{N}$ and

$$
\left\|f_{2}\right\|_{L^{1}\left(\mathbb{R}^{N}\right)} \leq \varepsilon, \quad\left\|f_{1}\right\|_{L^{\infty}\left(\mathbb{R}^{N}\right)} \leq K(\varepsilon) .
$$

Proof. Let

$$
f_{1, k}= \begin{cases}k, & \text { if } f \geq k \\ f, & \text { if }|f| \leq k \\ -k, & \text { if } f \leq-k\end{cases}
$$

and $f_{2, k}=f-f_{1, k}$. Then

$$
\left\|f_{2, k}\right\|_{L^{1}\left(\mathbb{R}^{N}\right)} \leq 2 \int_{\{|f| \geq k\}}|f| .
$$

Since

$$
|\{|f| \geq k\}|=\int_{\{|f| \geq k\}} 1 \leq \frac{1}{k}\|f\|_{L^{1}\left(\mathbb{R}^{N}\right)} \rightarrow 0, \quad \text { as } k \rightarrow \infty,
$$

invoking the dominated convergence theorem and (A.1), we conclude that $\left\|f_{2, k}\right\|_{L^{1}\left(\mathbb{R}^{N}\right)} \rightarrow 0$, as $k \rightarrow \infty$ and the conclusion follows.

Lemma A.4. Let $N \geq 1$. Assume that $f \in L^{p}\left(B\left(0, R_{0}\right)^{c}\right)$, for some $R_{0} \geq 0$ and $p \in[1, \infty)$. Then there exists a sequence $R_{n} \rightarrow \infty$ such that for all $s \in[0, N / p-N+1]$ we have

$$
R_{n}^{s} \int_{\partial B\left(0, R_{n}\right)}|f| d \sigma \leq \frac{K(p, N)}{\left(\ln R_{n}\right)^{p}}, \quad \text { as } n \rightarrow \infty,
$$

for some constant $K(p, N)>0$.

Proof. Since $f \in L^{p}\left(B\left(0, R_{0}\right)^{c}\right)$,

$$
\int_{R_{0}}^{\infty}\left(\int_{\partial B(0, r)}|f|^{p}\right) d r<\infty
$$

and thus there is a sequence $R_{n} \rightarrow \infty$, as $n \rightarrow \infty$, such that

$$
\int_{\partial B\left(0, R_{n}\right)}|f|^{p} \leq \frac{1}{R_{n} \ln \left(R_{n}\right)} .
$$

Then, using the Hölder inequality we obtain

$$
\int_{\partial B\left(0, R_{n}\right)}|f| \leq\left(K(N) R_{n}^{N-1}\right)^{1-1 / p} \frac{1}{\left(R_{n} \ln R_{n}\right)^{1 / p}},
$$

from where the result follows. 
Lemma A.5. Let $c \geq 0$ and $u \in C^{\infty}\left(\mathbb{R}^{N}\right) \cap U C\left(\mathbb{R}^{N}\right)$ be a solution of (TW . Assume that

$$
\underset{B(y, r)}{\operatorname{osc}} u \leq \frac{1}{8(1+c)(2 s+1)}
$$

for some $y \in \mathbb{R}^{N}, r>0$ and $s \geq 1$. Then

$$
\int_{B(y, r / 2)}|\nabla u|^{2(s+1)} \leq 4(1+c)^{2}\left(1+\frac{16}{r^{2}}\right) \int_{B(y, r)}|\nabla u|^{2 s} .
$$

Proof. The ideas of the proof are based on classical computations for elliptic equations with quadratic growth (see e.g. [31, [5, 28]). Therefore we only provide the main ideas, in order to show the dependence on $u, c, s$ and $N$ as stated. We set $B_{r} \equiv B(y, r)$ and $\eta \in C_{0}^{\infty}\left(B_{r}\right)$ a function such that $0 \leq \eta \leq 1$,

$$
|\nabla \eta| \leq \frac{4}{r} \text { on } B_{r} \quad \text { and } \quad \eta \equiv 1 \text { on } B_{r / 2} .
$$

Finally, we fix $w=|\nabla u|^{2}$, which is smooth by hypothesis, so that

$$
|\nabla w| \leq 2 w^{1 / 2}\left|D^{2} u\right| .
$$

We now divide the computations in several steps.

Step 1. If $\operatorname{osc}_{B_{r}} u \leq 1 / 4$, we have

$$
\int_{B_{r}} \eta^{2} w^{s+1} \leq \underset{B_{r}}{\operatorname{osc} u}\left(\int_{B_{r}}|\nabla \eta|^{2} w^{s}+\frac{2 s+1}{2} \int_{B_{r}} \eta^{2}\left|D^{2} u\right| w^{s-1}\right) .
$$

Indeed, since

$$
\int_{B_{r}} \eta^{2} w^{s+1}=\int_{B_{r}} \eta^{2} w^{s} \nabla(u-u(y)) \cdot \nabla u,
$$

integrating by parts and using (A.5), we deduce that

$$
\int_{B_{r}} \eta^{2} w^{s+1} \leq \underset{B_{r}}{\operatorname{osc}} u\left(2 \int_{B_{r}} \eta|\nabla \eta| w^{s+1 / 2}+(2 s+1) \int_{B_{r}} \eta^{2}\left|D^{2} u\right| w^{s}\right) .
$$

Using the elementary inequalities $2 a b \leq a^{2}+b^{2}$ and $a b \leq a^{2}+b^{2} / 4$ in the first and second integrals in the r.h.s. of (A.6), we obtain

$$
\left(1-\underset{B_{r}}{\operatorname{osc} u)} \int_{B_{r}} \eta^{2} w^{s+1} \leq \underset{B_{r}}{\operatorname{osc}} u\left(\int_{B_{r}}|\nabla \eta|^{2} w^{s}+\frac{(2 s+1)^{2}}{4} \int_{B_{r}} \eta^{2}\left|D^{2} u\right| w^{s-1}\right) .\right.
$$

Since $\operatorname{osc}_{B_{r}} u \leq 1 / 4$, we conclude Step 1

Step 2. We have

$$
\frac{1}{2} \int_{B_{r}} \eta^{2}\left|D^{2} u\right|^{2} w^{s-1} \leq 2 \int_{B_{r}}|\nabla \eta|^{2} w^{s}-\sum_{k=1}^{N} \int_{B_{r}} \partial_{k}(\Delta u) \cdot \partial_{k} u \eta^{2} w^{s-1} .
$$

Let $k \in\{1, \ldots, N\}$ and $\phi_{k}=\eta^{2} w^{s-1} \partial_{k} u \in C_{0}^{\infty}\left(B_{r}\right)$. On one hand, integrating by parts,

$$
\sum_{j=1}^{N} \int_{B_{r}} \partial_{j k}^{2} u \cdot \partial_{j} \phi_{k}=-\int_{B_{r}} \partial_{k}(\Delta u) \cdot \phi_{k}
$$

On the other hand, using that

$$
\partial_{j} w=2 \sum_{k=1}^{N} \partial_{k} u \cdot \partial_{j k}^{2} u
$$


and developing the term $\partial_{j} \phi_{k}$,

$$
\begin{aligned}
\sum_{j, k=1}^{N} \int_{B_{r}} \partial_{j k}^{2} u \cdot \partial_{j} \phi_{k}= & \int_{B_{r}} \eta^{2}\left|D^{2} u\right|^{2} w^{s-1}+2 \sum_{j, k=1}^{N} \int_{B_{r}} \eta \partial_{j} \eta w^{s-1} \partial_{k} u \cdot \partial_{j k}^{2} u \\
& +\frac{s-1}{2} \int_{B_{r}} \eta^{2} w^{s-2}|\nabla w|^{2}
\end{aligned}
$$

Then the conclusion of this step follows combining (A.7) and (A.8), noticing that the last integral in the r.h.s. of (A.8) is nonnegative, and that

$$
2 \sum_{j, k=1}^{N}\left|\eta \partial_{j} \eta w^{s-1} \partial_{j k}^{2} u \cdot \partial_{k} u\right| \leq 2 \sum_{j, k=1}^{N} \eta\left|\partial_{j k}^{2} u\right| w^{\frac{s-1}{2}} \cdot\left|\partial_{j} \eta \| \partial_{k} u\right| w^{\frac{s-1}{2}} \leq \frac{1}{2} \eta^{2}\left|D^{2} u\right|^{2} w^{s-1}+2|\nabla \eta|^{2} w^{s} .
$$

Step 3. For all $\delta>0$, we have

$$
\sum_{j=1}^{N}\left|\partial_{j} \Delta u \cdot \partial_{j} u\right| \leq \delta(c+1)\left|D^{2} u\right|^{2}+\left(c+\frac{c}{\delta}+4\right) w+(1+c) w^{2} .
$$

Using $\left(\mathrm{TW}_{c}\right)$ and the fact that $|u|=1$, it is simple to check that

$$
\sum_{j=1}^{N}\left|\partial_{j} \Delta u \cdot \partial_{j} u\right| \leq 2 w\left|D^{2} u\right|+w^{2}+4 w+2 c w^{3 / 2}+2 c\left|D^{2} u\right| w^{1 / 2} .
$$

By combining with the fact that $2 a b \leq \delta a^{2}+\delta^{-1} b^{2}$, for all $\delta>0$, we finish Step 3 ,

Step 4.

$$
\frac{1}{4} \int_{B_{r}} \eta^{2}\left|D^{2} u\right|^{2} w^{s-1} \leq 2 \int_{B_{r}}|\nabla \eta|^{2} w^{s}+\left(4 c^{2}+5 c+4\right) \int_{B_{r}} \eta^{2} w^{s}+(c+1) \int_{B_{r}} \eta^{2} w^{s+1} .
$$

Step 4 follows immediately from Steps 2 and 3 taking $\delta=(4(c+1))^{-1}$.

Now we are in position to finish the proof of Lemma A.5. In fact, by combining Steps 1 and 4 we are led to

$$
\begin{aligned}
\left(1-4(c+1)(2 s+1) \underset{B_{r}}{\operatorname{osc} u)} \int_{B_{r}} \eta^{2} w^{s+1} \leq\right. & \underset{B_{r}}{\operatorname{osc} u}\left((8 s+5) \int_{B_{r}}|\nabla \eta|^{2} w^{s}+\right. \\
& \left.2\left(4 c^{2}+5 c+4\right)(2 s+1) \int_{B_{r}} \eta^{2} w^{s}\right) .
\end{aligned}
$$

Also, we see that (A.2) implies that

$$
1 / 2 \leq\left(1-4(c+1)(2 s+1) \underset{B_{r}}{\operatorname{osc} u)}\right.
$$

and that

$$
2 \underset{B_{r}}{\operatorname{osc} u} u \cdot \max \left\{8 s+5,2\left(4 c^{2}+5 c+4\right)(2 s+1)\right\} \leq \frac{2\left(4 c^{2}+5 c+4\right)}{4(1+c)} \leq 2\left(1+c^{2}\right) .
$$

By combining with (A.4), we conclude (A.3).

\section{References}

[1] F. Béthuel, P. Gravejat, and J.-C. Saut. Existence and properties of travelling waves for the GrossPitaevskii equation. In A. Farina and J.-C. Saut, editors, Stationary and time dependent GrossPitaevskii equations. Wolfgang Pauli Institute 2006 thematic program, January-December, 2006, Vienna, Austria, volume 473 of Contemporary Mathematics, pages 55-104. American Mathematical Society.

[2] F. Béthuel, P. Gravejat, and J.-C. Saut. Travelling waves for the Gross-Pitaevskii equation. II. Comm. Math. Phys., 285(2):567-651, 2009. 
[3] F. Béthuel, G. Orlandi, and D. Smets. Vortex rings for the Gross-Pitaevskii equation. J. Eur. Math. Soc. (JEMS), 6(1):17-94, 2004.

[4] J. L. Bona and Y. A. Li. Decay and analyticity of solitary waves. J. Math. Pures Appl. (9), 76(5):377-430, 1997.

[5] H.-J. Borchers and W. D. Garber. Analyticity of solutions of the $O(N)$ nonlinear $\sigma$-model. Comm. Math. Phys., 71(3):299-309, 1980.

[6] H. Brezis and J.-M. Coron. Multiple solutions of $H$-systems and Rellich's conjecture. Comm. Pure Appl. Math., 37(2):149-187, 1984.

[7] D. Chiron and M. Mariş. Traveling waves for nonlinear Schrödinger equations with nonzero conditions at infinity, II. Preprint arXiv:1203.1912.

[8] A. de Bouard and J.-C. Saut. Symmetries and decay of the generalized Kadomtsev-Petviashvili solitary waves. SIAM J. Math. Anal., 28(5):1064-1085, 1997.

[9] A. de Laire. Non-existence for travelling waves with small energy for the Gross-Pitaevskii equation in dimension $N \geq 3$. C. R. Math. Acad. Sci. Paris, 347(7-8):375-380, 2009.

[10] A. de Laire. Global well-posedness for a nonlocal Gross-Pitaevskii equation with non-zero condition at infinity. Comm. Partial Differential Equations, 35(11):2021-2058, 2010.

[11] A. Farina. From Ginzburg-Landau to Gross-Pitaevskii. Monatsh. Math., 139(4):265-269, 2003.

[12] M. Giaquinta and G. Modica. Almost-everywhere regularity results for solutions of nonlinear elliptic systems. Manuscripta Math., 28(1-3):109-158, 1979.

[13] V. Girault and P.-A. Raviart. Finite element methods for Navier-Stokes equations, volume 5 of Springer Series in Computational Mathematics. Springer-Verlag, Berlin, 1986. Theory and algorithms.

[14] P. Gravejat. Decay for travelling waves in the Gross-Pitaevskii equation. Ann. Inst. H. Poincaré Anal. Non Linéaire, 21(5):591-637, 2004.

[15] P. Gravejat. Asymptotics for the travelling waves in the Gross-Pitaevskii equation. Asymptot. Anal., $45(3-4): 227-299,2005$.

[16] P. Gravejat. First order asymptotics for the travelling waves in the Gross-Pitaevskii equation. Adv. Differential Equations, 11(3):259-280, 2006.

[17] B. Guo and S. Ding. Landau-Lifshitz equations, volume 1 of Frontiers of Research with the Chinese Academy of Sciences. World Scientific Publishing Co. Pte. Ltd., Hackensack, NJ, 2008.

[18] S. Gustafson, K. Nakanishi, and T.-P. Tsai. Scattering for the Gross-Pitaevskii equation. Math. Res. Lett., 13(2-3):273-285, 2006.

[19] S. Gustafson, K. Nakanishi, and T.-P. Tsai. Scattering theory for the Gross-Pitaevskii equation in three dimensions. Commun. Contemp. Math., 11(4):657-707, 2009.

[20] S. Gustafson and J. Shatah. The stability of localized solutions of Landau-Lifshitz equations. Comm. Pure Appl. Math., 55(9):1136-1159, 2002.

[21] F. B. Hang and F. H. Lin. Static theory for planar ferromagnets and antiferromagnets. Acta Math. Sin. (Engl. Ser.), 17(4):541-580, 2001.

[22] F. Hélein. Régularité des applications faiblement harmoniques entre une surface et une variété riemannienne. C. R. Acad. Sci. Paris Sér. I Math., 312(8):591-596, 1991.

[23] F. Hélein. Harmonic maps, conservation laws and moving frames, volume 150 of Cambridge Tracts in Mathematics. Cambridge University Press, Cambridge, second edition, 2002. Translated from the 1996 French original, With a foreword by James Eells. 
[24] L. Hörmander. The analysis of linear partial differential operators I. Classics in Mathematics. Springer-Verlag, Berlin, 2003.

[25] A. Hubert and R. Schäfer. Magnetic domains: the analysis of magnetic microstructures. Springer, 1998.

[26] C. A. Jones, S. J. Putterman, and P. H. Roberts. Motions in a Bose condensate V. Stability of solitary wave solutions of non-linear Schrödinger equations in two and three dimensions. J. Phys. A, Math. Gen., 19(15):2991-3011, 1986.

[27] C. A. Jones and P. H. Roberts. Motions in a Bose condensate IV. Axisymmetric solitary waves. J. Phys. A, Math. Gen., 15(8):2599-2619, 1982.

[28] J. Jost. Riemannian geometry and geometric analysis. Universitext. Springer-Verlag, Berlin, fifth edition, 2008.

[29] A. M. Kosevich, B. A. Ivanov, and A. S. Kovalev. Magnetic solitons. Physics Reports, 194(3-4):117$238,1990$.

[30] P. Koskela, J. J. Manfredi, and E. Villamor. Regularity theory and traces of $\mathcal{A}$-harmonic functions. Trans. Amer. Math. Soc., 348(2):755-766, 1996.

[31] O. A. Ladyzhenskaya and N. N. Ural'tseva. Linear and quasilinear elliptic equations. Translated from the Russian by Scripta Technica, Inc. Translation editor: Leon Ehrenpreis. Academic Press, New York, 1968.

[32] L. Landau and E. Lifshitz. On the theory of the dispersion of magnetic permeability in ferromagnetic bodies. Phys. Z. Sowjetunion, 8:153-169, 1935.

[33] F. Lin and J. Wei. Traveling wave solutions of the Schrödinger map equation. Comm. Pure Appl. Math., 63(12):1585-1621, 2010.

[34] M. Mariş. Nonexistence of supersonic traveling waves for nonlinear Schrödinger equations with nonzero conditions at infinity. SIAM J. Math. Anal., 40(3):1076-1103, 2008.

[35] M. Mariş. Traveling waves for nonlinear Schrödinger equations with nonzero conditions at infinity. Ann. of Math. (2), 178(1):107-182, 2013.

[36] H. Mikeska. Solitons in one-dimensional magnets with various symmetries. In J. Bernasconi and T. Schneider, editors, Physics in one dimension. Proceedings of an international conference,Fribourg, Switzerland, August 25-29, 1980, pages 153-156. Springer Series in Solid-State Sciences, 23. BerlinHeidelberg-New York: Springer-Verlag, 1981.

[37] R. Moser. Partial regularity for harmonic maps and related problems. World Scientific Publishing Co. Pte. Ltd., Hackensack, NJ, 2005.

[38] A. Nahmod, A. Stefanov, and K. Uhlenbeck. On Schrödinger maps. Comm. Pure Appl. Math., 56(1):114-151, 2003.

[39] K. Nakamura and T. Sasada. Quantum kink in the continuous one-dimensional Heisenberg ferromagnet with easy plane: a picture of the antiferromagnetic magnon. J. Phys. C: Solid State Phys., 15(28):L1013-L1017, 1982.

[40] N. Papanicolaou and P. N. Spathis. Semitopological solitons in planar ferromagnets. Nonlinearity, 12(2):285-302, 1999.

[41] B. Piette and W. J. Zakrzewski. Localized solutions in a two-dimensional Landau-Lifshitz model. Physica D: Nonlinear Phenomena, 119(3-4):314-326, 1998.

[42] X. Pu and B. Guo. A note on vortex solutions of Landau-Lifshitz equation. Math. Methods Appl. Sci., 33(7):874-879, 2010.

[43] T. Rivière. Everywhere discontinuous harmonic maps into spheres. Acta Math., 175(2):197-226, 1995. 
[44] T. Sasada. Magnons, solitons, and a critical field in the heisenberg ferromagnetic chain with easyplane anisotropy. Journal of the Physical Society of Japan, 51(8):2446-2449, 1982.

[45] G. Stampacchia. Le problème de Dirichlet pour les équations elliptiques du second ordre à coefficients discontinus. Ann. Inst. Fourier (Grenoble), 15(fasc. 1):189-258, 1965.

[46] P. Topping. The optimal constant in Wente's $L^{\infty}$ estimate. Comment. Math. Helv., 72(2):316-328, 1997.

[47] J. Wei and W. Yao. Asymptotic axisymmetry of the subsonic traveling waves to the Gross-Pitaevskii equation. Commun. Contemp. Math., 13(6):1095-1104, 2011.

[48] H. C. Wente. An existence theorem for surfaces of constant mean curvature. J. Math. Anal. Appl., 26:318-344, 1969. 